\title{
A Quantitative Comparison of Multiple Access Control Protocols for Wireless ATM
}

\author{
Yu-Kwong Kwok, Member, IEEE, and Vincent K. N. Lau
}

\begin{abstract}
The multiple access control (MAC) problem in a wireless network has intrigued researchers for years. For a broad-band wireless network such as wireless ATM, an effective MAC protocol is very much desired because efficient allocation of channel bandwidth is imperative in accommodating a large user population with satisfactory quality of service. Indeed, MAC protocols for a wireless ATM network in which user traffic requirements are highly heterogeneous (classified into CBR, VBR, and ABR), are even more intricate to design. Considerable research efforts expended in tackling the problem have resulted in a myriad of MAC protocols. While each protocol is individually shown to be effective by the respective designers, it is unclear how these different protocols compare against each other on a unified basis. In this paper, we quantitatively compare seven recently proposed TDMA-based MAC protocols for integrated wireless data and voice services. We first propose a taxonomy of TDMA-based protocols, from which we carefully select seven protocols, namely SCAMA, DTDMA/VR, DTDMA/PR, DQRUMA, DPRMA, DSA + +, and PRMA/DA, such that they are devised based on rather orthogonal design philosophies. The objective of our comparison is to highlight the merits and demerits of different protocol designs.
\end{abstract}

Index Terms-Adaptive protocol, FDD, multiple access control, TDMA, wireless ATM.

\section{INTRODUCTION}

$\mathbf{T}$ HE classical design of a channel multiple access control (MAC) protocol is to arbitrate and statistically multiplex the transmission requests of multiple uncoordinated users and allocate transmission bandwidth to the users in a fair manner. Notorious examples include the ALOHA protocol for a packet radio network and the CSMA/CD protocol for a wired local area network. The key feature of the classic design is that all users are homogeneous-they have the same traffic characteristics. However, in our study, we consider a wireless ATM system for integrated isochronous and bursty services [15] (see Fig. 1), for which an effective and intelligent MAC protocol is particularly desired due to the sharing of the precious bandwidth by a dynamically changing population of users with various traffic demands. A MAC protocol for wireless ATM distinguishes itself from other MAC protocols in that various mechanisms are required to handle the diverse traffic demands of different services such as constant bit rate (CBR), variable bit rate (VBR), and available bit rate (ABR)

Manuscript received January 31, 2000; revised August 8, 2000. This work was supported by Seed Grants from HKU URC under contract numbers 10203010 and 10203413 and by Earmarked Grants from the Hong Kong Research Grants Council under contract numbers HKU 7124/99E and HKU 7024/00E.

The authors are with the Department of Electrical and Electronic Engineering The University of Hong Kong, Pokfulam Road, Hong Kong.

Publisher Item Identifier S 0018-9545(01)03961-5.
[15]. CBR traffic such as voice telephony, VBR traffic such as video conferencing, and $\mathrm{ABR}$ traffic such as file data have very different service requirements in terms of delay and loss tolerance and throughput. Multiplexing these diverse services harmoniously such that reasonable quality of service (QoS) is maintained while at the same time maximizing the utilization of the precious channel bandwidth is a daunting and challenging task. Thus, while traditional ALOHA-type MAC protocols can handle homogeneous traffic efficiently, different techniques are needed for a wireless ATM system. As such, there are a plethora of efficient MAC protocols reported in the literature that are specially designed for wireless ATM [2]-[4], [7]-[9], [13], [14], [16]. In general, there are four aspects in characterizing these MAC protocols.

- Request Mechanism: The mechanism of receiving user requests critically affects the performance of a MAC protocol. For example, in some contention based protocols, too much contention (e.g., a large number of active users) will result in system instability such that users keep on contending without success due to excessive collisions. Under such a thrashing situation, most of the information slots are not used. Different protocols employ various techniques to combat this problem.

- Slots Allocation: In most MAC protocols, information slots are assigned on a first-come first-serve basis and can be reserved in subsequent frames if the user is a voice terminal. However, some recently proposed protocols employ more intelligent approaches to further enhance the channel utilization.

- Frame Structure: Traditionally, the frame is of a static structure. That is, for example, there is a fixed portion of the frame dedicated for receiving transmission requests, while the remaining portion is for information slots. A major merit of a static frame structure is the ease of implementation and is energy efficient for the mobiles, which do not need to listen to the channel all the time. Some other protocols, however, employ a dynamic frame structure, with the objective to utilize the bandwidth more efficiently.

- Performance: The capacity of the network and QoS depend critically on the performance of the MAC protocol in terms of packet dropping rate, delay, throughput, and utilization.

A scrutiny of the above four aspects reveals a large design space for MAC protocols. Indeed, based on these four dimensions, we introduce a simple taxonomy of recent MAC protocols suggested in the literature for wireless ATM. With the taxonomy, seven MAC protocols, designed based on radically dif- 


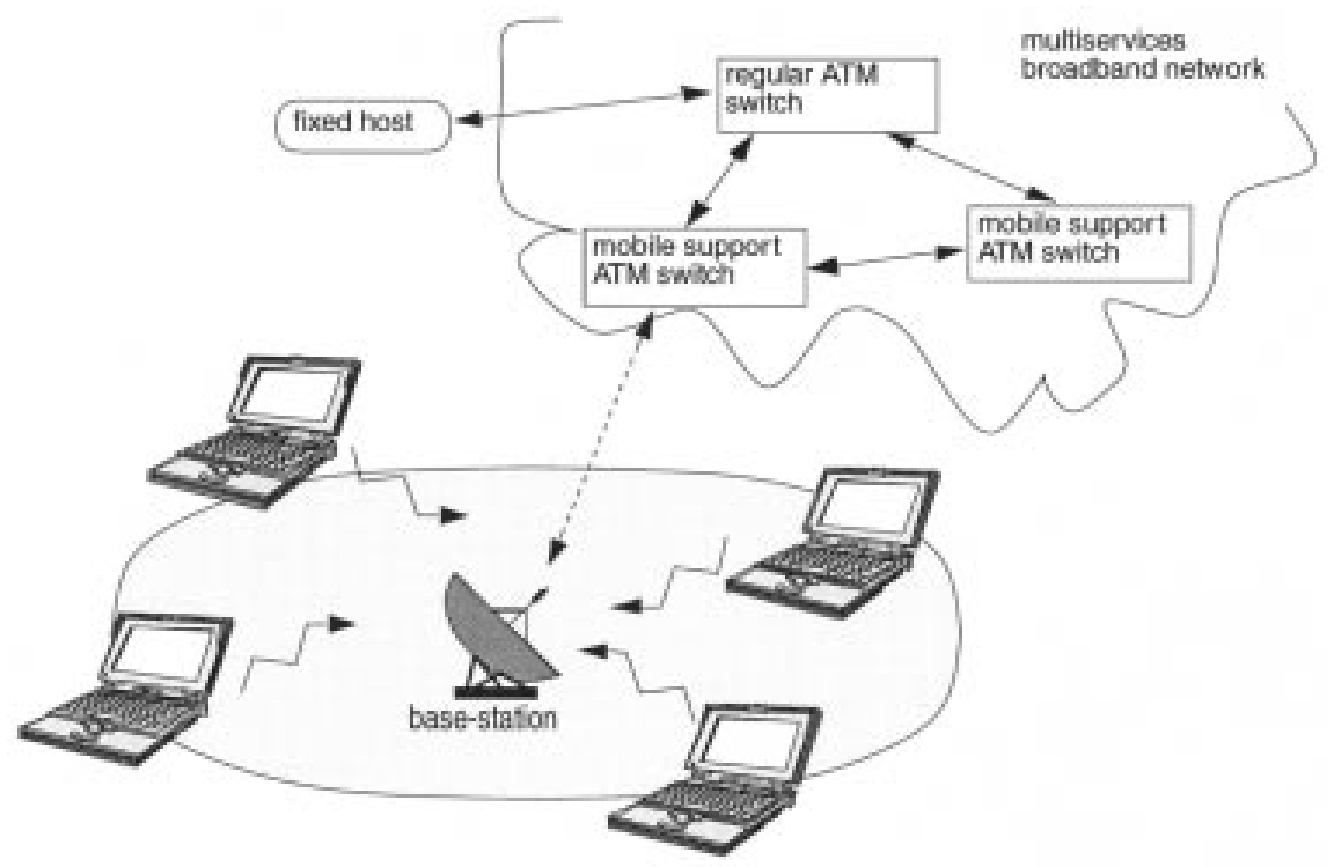

Fig. 1. Broad-band mobile computing environment based on wireless ATM.

ferent philosophies, are selected for an extensive performance comparison. The protocols chosen are as follows.

- DQRUMA [7]: distributed queueing request update multiple access, a protocol that employs a novel piggybacking request update mechanism.

- DSA ++ [13]: dynamic slot assignment, a protocol that is based on an intelligent slots allocation and scheduling mechanism.

- PRMA/DA [9]: packet reservation multiple access with dynamic allocation, a protocol that employs a novel dynamic frame structure.

- DTDMA/PR [14]: dynamic TDMA with piggyback reservation, a protocol that uses a dynamically adjust partitioning of information slots assigned for real time (i.e., CBR or VBR) and nonreal time (i.e., ABR) users.

- DTDMA/VR [8]: dynamic TDMA with variable rate transmission, a protocol that uses an adaptive physical layer.

- DPRMA [4]: dynamic packet reservation multiple access, a protocol that uses a rate-controlled allocation strategy.

- SCAMA [12]: synergistic channel-adaptive multiple access, an adaptive protocol which works closely with the underlying physical layer in that through observing the channel state information (CSI) of each mobile user, the SCAMA protocol first segregates a set of users with good CSI from requests gathered in the request contention phase of an uplink frame. The SCAMA protocol then judiciously allocates information time slots to the users according to their traffic types, CSI, urgency, and throughput, which are collectively represented by a novel priority function.

We have implemented all the seven protocols on a common simulation platform, from which extensive performance results are obtained. The protocols are evaluated for test cases with and without request queues, which store transmission requests that survive the contention but fail to get assigned information slots.
Three performance metrics, namely cell-loss rate, ABR data delay, and ABR data throughput, are considered. The balance of this paper is as follows. In Section II, we present a detailed scrutiny of the TDMA-based MAC problem, with the help of a simple taxonomy. The selected protocols are then described in detail in Section III. Section IV contains the performance results and our interpretations. The final section provides some concluding remarks.

\section{MAC PROTOCOLS FOR WIRELESS ATM}

As tetherless communication and computing becomes more and more ubiquitous, one of the most important issues in a cellular wireless network is how to effectively manage the precious bandwidth so as to support a large and dynamic user population with a reasonable level of service quality. The abundant bandwidth available in a broad-band wireless systems does not help to alleviate the problem because the user population size will for sure increase proportionately. Thus, bandwidth management problems, having baffled researchers for years, continue to be a rich land of research. The goal of a MAC protocol is to judiciously arbitrate the transmission requests of multiple uncoordinated users and assign transmission bandwidth to the users. While the MAC problem also exists in wired networks, a MAC protocol in a wireless communication system is particularly important due to the sharing of the precious bandwidth by a dynamically changing population of users with various traffic demands. Indeed, the dynamic nature of users population, both spatially and temporally, makes the capacity of the network and quality of service depend critically on the performance of the MAC protocol in terms of packet dropping rate, delay, and throughput.

Fig. 2 depicts a partial taxonomy of TDMA based MAC protocols for wireless ATM considered in our study. In a TDMAbased protocol, time is divided into slots, which are grouped into 


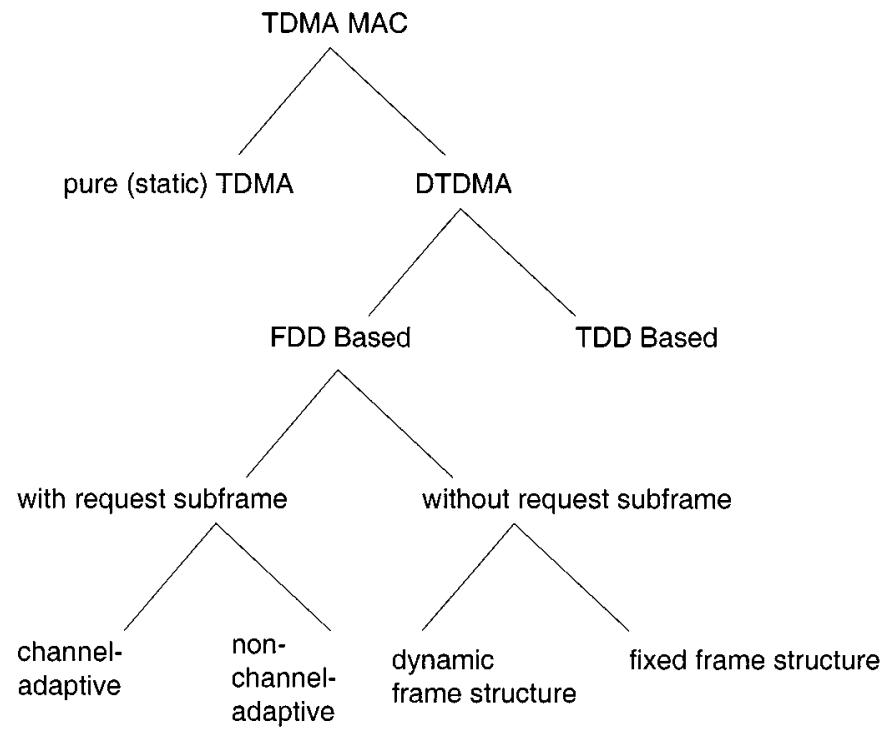

Fig. 2. A taxonomy of TDMA-based MAC protocols for wireless ATM.

frames. The slots, more specifically the information slots, are for users to transmit information packets. In general, the users contend for information slots on a frame-by-frame basis. There are two types of TDMA-based protocols: pure TDMA and $d y$ namic TDMA (DTDMA). In a pure TDMA protocol, the slots assignment, determined statically, is fixed throughout the communication process without regard to the actual requirements of the users. Thus, even if a user does not have any information packet to send, the assigned time slot is still occupied and, therefore, is wasted. In view of the poor utilization and inflexibility, pure TDMA protocols are not used in the cellular wireless networks considered in this paper, due to the dynamic users population involved. DTDMA protocols can be classified as time division duplex (TDD) or frequency division duplex (FDD) based. A TDD based protocol (e.g., the MASCARA protocol [2]) can exploit the asymmetry between the uplink (heavily loaded) and downlink (lightly loaded) communications in that the bandwidth for downlink communication can be converted for uplink communication when mobile traffic load is high. However, a TDD based system suffers from the long and irregular delay in both the uplink and downlink communications. A FDD scheme, by contrast, can exploit the almost instantaneous acknowledgment (i.e., short propagation delay) in both uplink and downlink transmissions and, as such, the interface design is also simpler. We focus on FDD schemes only in this paper.

Time on the FDD based uplink channel is also divided into a contiguous sequence of TDMA frames, which are subdivided into request slots and information slots. The information slots are sometimes further classified into CBR/VBR and ABR slots. There are two types of packets being transmitted in the channel, namely the request packet and the information packet. A request packet is used for the request of information slot (for carrying CBR, VBR, or ABR data cells). However, most earlier protocols do not distinguish slots for transmitting request or information data, as in the classical PRMA protocol [5] [see Fig. 3(a)]. Indeed, these protocols use also the information slot for requests contention and, as such, the bandwidth utilization can be low if traffic load is high. Some more recently proposed protocols par-

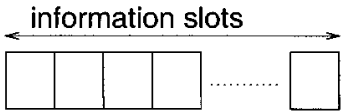

(a)

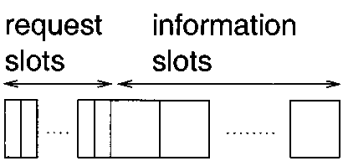

(b)

Fig. 3. Frame structures of (a) the classical PRMA protocol and (b) a generic DTDMA protocol.

tition the TDMA frame into two parts: request subframe and information subframe, as shown in Fig. 3(b). The motivation of this approach is that a request packet often includes only very small of amount of information, namely the origin and the destination addresses, and is therefore usually much shorter than a information packet. The request subframe, therefore, can be operated with a much shorter slot duration using the slotted-ALOHA protocol.

MAC protocols can be further classified into two categories: dynamic frame structure and fixed frame structure. In the former category, the frame structure, in terms of frame length or frame format, is varied over time. The objective is to optimize the bandwidth usage for requesting and information transmission. Finally, in the fixed frame structure category, some recently proposed protocols (e.g., DTDMA/VR and SCAMA) use a variable-throughput frame supported by a channel adaptive physical layer. Such channel adaptive protocols are motivated by the fact that, in general, previous protocols attempt to accommodate more data users, which do not impose constraints on data delay, by exploiting the silence gaps of the CBR/VBR users, which require bounded-delay packet transmission and hence, enjoy a higher transmission priority than $\mathrm{ABR}$ users in that reservation is allowed for the former but not the latter. However, while sophisticated slot allocation strategies with articulated frame structures are proposed in these methods, most of them considers the effect of burst channel errors on protocol performance, let alone the investigation of exploiting the error characteristics to enhance performance. Essentially, these previous protocols are designed and analyzed based on the assumption that packet transmission through the wireless channel is error free. Indeed, because the geographically scattered mobile users inevitably suffer from different degrees of fading and shadowing effects, a common drawback of previous MAC protocols is that they assume the underlying physical layer always delivers a constant throughput, and as such, they may not be able to effectively utilize the precious bandwidth when the channel condition is swiftly varying among different users.

\section{PROTOCOL DESCRIPTIONS}

From the taxonomy shown in Fig. 2, seven recent protocols are selected for our quantitative comparison, with the objective to highlight the merits and demerits of different protocol designs. The protocols selected and their distinctive characteristics are listed in Table I. For completeness, the operations of these protocols are outlined below. Notice that the time complexity of each protocol refers to the worst case number of steps required for the protocol to schedule one frame and $n$ is the number of re- 
TABLE I

PROTOCOL CHARACTERISTICS

\begin{tabular}{l|cccc}
\hline Protocol & With Request Subframe & Dynamic Frame & Highest Priority & Complexity \\
\hline DQRUMA & No & Yes & - & $O(n)$ \\
DSA++ & Yes & No & CBR & $O(n \log n)$ \\
PRMA/DA & No & Yes & CBR & $O(n)$ \\
DTDMA/PR & Yes & No & CBR & $O(n)$ \\
DTDMA/VR & Yes & No & - & $O(n)$ \\
DPRMA & No & No & VBR & $O(n)$ \\
SCAMA & Yes & No & CBR/VBR & $O(n \log n)$ \\
\hline
\end{tabular}

quests to be handled within one frame (in the worst case, which is a very rare situation, $n$ may be as large as the total number of users in the system). The reader is referred to the respective references for more detailed information.

\section{A. DQRUMA}

The DQRUMA protocol proposed by Karol et al. [7] is a well-known MAC protocol for wireless ATM. In simple terms, being an FDD protocol, DQRUMA works by dividing the uplink frame into two sections with the first section for requests contention, whereas the second section for information data transmissions. A nice and novel feature of the DQRUMA protocol is the use of a piggyback reservation field, with which a VBR user can update the traffic slot demand without the need of an extra contention. Another novel feature of the DQRUMA protocol is that if needed, the base station can convert one information slot into a number of request slots so as to alleviate the contention. However, despite that the dynamic frame structure and piggybacking mechanism are useful for better channel utilization, the DQRUMA protocol suffers from the lack of an intelligent slots allocation strategy. Thus, the QoS of different users (CBR, VBR, and $\mathrm{ABR}$ ) are not optimized.

\section{B. $D S A++$}

The DSA ++ protocol proposed by Petras and Krämling [13]. The DSA++ protocol employs a variable-length frame structure called a signaling burst, in which a mobile user can reserve information time slots in the uplink frame by using the reservation slots in the uplink reservation phase. To handle the heterogeneous QoS requirements, the assignment of information time slots is performed by a scheduler in the base station, which uses a priority function composed of the number of pending cells and the deadlines. The priorities are assigned such that priorities between classes are constrained by the relationship: $\mathrm{CBR}>\mathrm{VBR}$ $>$ ABR. The strength of the DSA ++ protocol is its comprehensive prioritization process. Moreover, it should be noted that the underlying scheduling policy of the DSA ++ protocol (and MAC protocols in general) can also play an important role in determining the performance of the system [13]. Indeed, an intelligent scheduling approach can further enhance the accuracy of the prioritization process. However, in this study, we focus on the MAC layer only.

\section{PRMA/DA}

The PRMA/DA proposed by Kim and Widjaja [9] is an enhanced version of the PRMA protocol [5]. Basically, the PRMA protocol is extended to allow VBR users to make rate-based reservations. A novel feature of the PRMA/DA protocol is that the dynamic framing mechanism, in which the base station adjusts the relative proportions of request slots and information slots according to the extent of request contention. For example, if the base station detects a collision in a certain request slot, it infers that there are at least two mobiles contending for a request slot and, as such, in the next frame, one more request slot will be added. On the other hand, if the base station finds that a certain request slot is unused, one request slot will be converted to an information slot in the next frame. This dynamic framing mechanism can enhance the stability as well as utilization of the system. However, since a full information slot is used for requesting also, the bandwidth wastage can be significant.

\section{DTDMA/PR}

Similar to DQRUMA, the DTDMA/PR proposed by Qiu et al. [14] also allows VBR users to update time slots requirements using a piggyback field in an uplink information slot. A distinctive feature of DTDMA/PR is that the information slots are classified as long-term and short-term reservable. Specifically, long-term slots are for CBR and VBR users, while short-term ones are for $\mathrm{ABR}$ users.

\section{E. DTDMA/VR}

The DTDMA/VR [8] protocol employs a static frame structure in that the time frame is divided into two parts: $N_{r}$ request slots and $N_{i}$ information slots. Specifically, a traditional slots assignment strategy is used in that whenever a request is successfully received in the request phase, information slots, if any, are immediately assigned to the requests. The DTDMA/VR protocol also considers the effect of burst errors by employing a channel adaptive physical layer. However, in contrast to the proposed SCAMA protocol, the DTDMA/VR does not consider the synergistic interaction between the MAC and physical layers and therefore, it is not a fully adaptive protocol. In other words, the MAC layer is not aware of the current situation in the physical layer in the process of bandwidth allocation. However, with the help of the channel-adaptive physical layer, DTDMA/VR has twice the average offered throughput compared to previous protocols [11].

\section{F. DPRMA}

The DPRMA protocol proposed by Dyson and Haas [4]. Information time slots within a DPRMA frame are partitioned among the mobiles based on the amount of slots required by each user. Mobile users may reserve a number of slots within a frame or even slots in alternating frames, as long as there is 


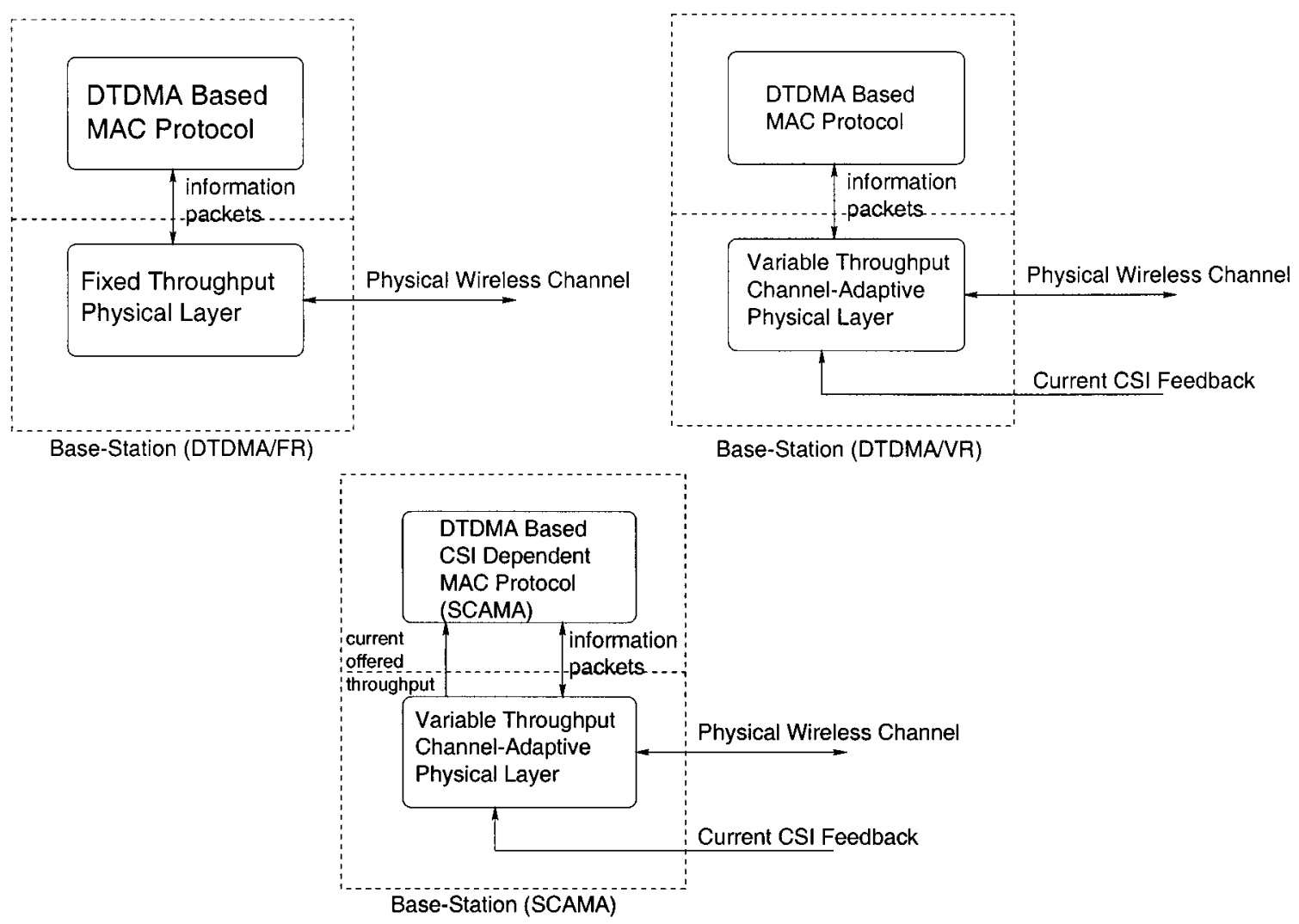

Fig. 4. Conceptual models of the physical and MAC layers.

frame capacity. However, if capacity is not enough, users are required to share the deficit proportionately. In contrast to the DSA ++ protocol, DPRMA gives a slightly higher priority to VBR users. Specifically, the DPRMA protocol uses a rate-controlled approach in that slots allocation is based on the relative bandwidth requirements among the users. However, when traffic load is high so that there are not enough information slots, the users are required to share the deficit proportionately according to their rate requirements.

\section{G. SCAMA}

The SCAMA is recently proposed by us [12] and is based on a novel concept-exploiting the synergy between two protocol layers instead of strictly following the traditional information hiding protocol design paradigm. Fig. 4 highlights the differences in the designs among a traditional fixed throughput MAC protocol (denoted generically as DTDMA/FR), DTDMA/VR, and SCAMA. Because the SCAMA protocol is highly adaptive and is the first of its kind, we describe its operation in more detail below. To obtain more information about the functionality and analysis of the SCAMA protocol, the reader is referred to [12].

1) Wireless Channel Model: The wireless communication environment considered in this paper is the reverse-link situation of a wireless system where a number of mobile terminals contend to transmit ATM cells to an ATM server. The wireless link between a mobile ATM terminal and the ATM server is characterized by two components, namely the microscopic fading component and the macroscopic shadowing component [1]. Microscopic fading is caused by the superposition of mul- tipath components and is therefore fluctuating in a fast manner (on the order of a few milliseconds). Macroscopic shadowing is caused by terrain configuration or obstacles and is fluctuating in a relatively slow manner (on the order of 1 to $2 \mathrm{~s}$ ). To illustrate, a sample of measured fading signal is shown in Fig. 5.

Let $c(t)$ be the combined channel fading which is given by

$$
c(t)=c_{l}(t) c_{s}(t)
$$

where $c_{l}(t)$ and $c_{s}(t)$ are the long-term macroscopic and short-term microscopic fading components, respectively. Both $c_{s}(t)$ and $c_{l}(t)$ are random processes with coherent time on the order of a few milliseconds and seconds, respectively.

a) Short-term microscopic fading: Without loss of generality, we assume $\mathcal{E}\left[c_{s}^{2}(t)\right]=1$ where $\mathcal{E}[]$ denotes the expected value of a random variable. The probability distribution of $c_{s}(t)$ follows the Rayleigh distribution which is given by

$$
f_{c_{s}}\left(c_{s}\right)=c_{s} \exp \left(-\frac{c_{s}^{2}}{2}\right)
$$

In this paper, we assume the maximum mobile speed is $40 \mathrm{~km} / \mathrm{h}$ and, hence, the Doppler spread [18], $f_{d} \approx 50 \mathrm{~Hz}$. It follows that the coherent time $T_{c}$ is approximately given by

$$
T_{c} \approx \frac{1}{f_{d}}
$$

which is about $20 \mathrm{~ms}$. 


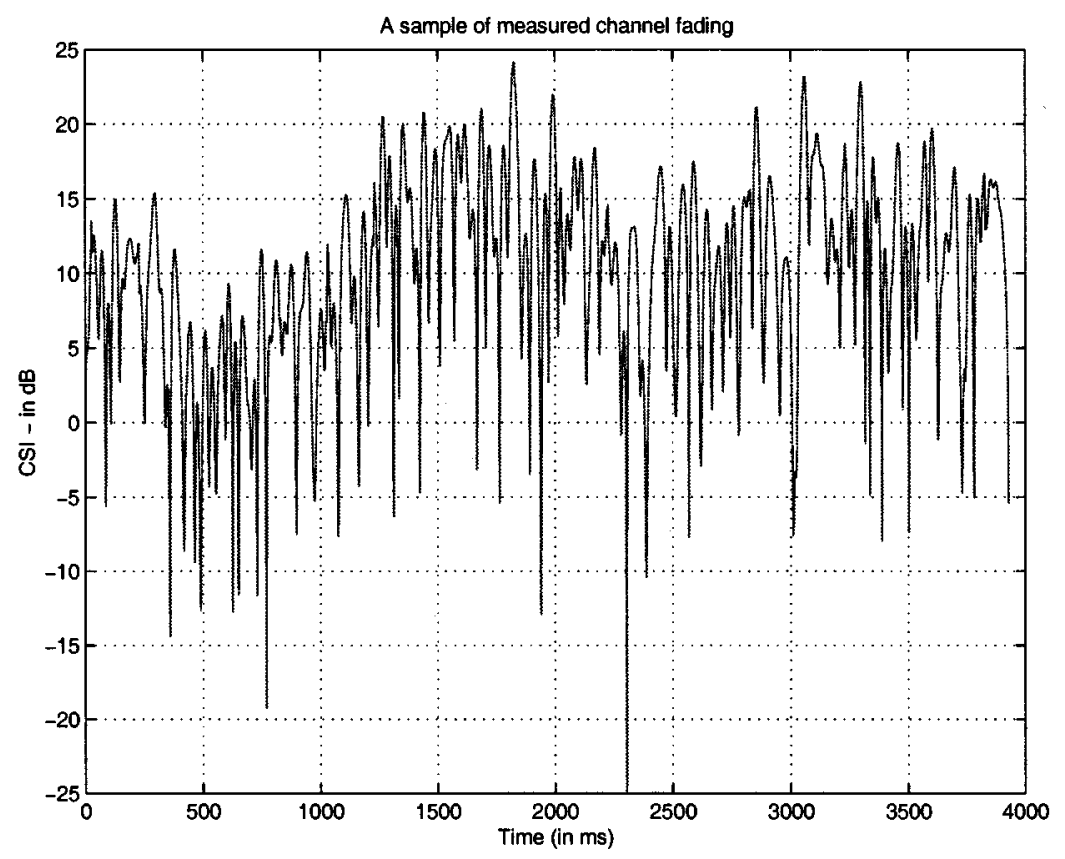

Fig. 5. A sample of channel fading with microscopic fading superimposed on macroscopic shadowing.

b) Long-term macroscopic shadowing: The long-term fading component, $c_{l}(t)$, is also referred to as the local mean [18], which, as shown by field test measurement, obeys the log-normal distribution $f_{c_{l}}\left(c_{l}\right)$. That is

$$
f_{c_{l}}\left(c_{l}\right)=\frac{4.34}{\sqrt{2 \pi} \sigma_{l} c_{l}} \exp \left(-\frac{\left(c_{l}(d B)-m_{l}\right)^{2}}{2 \sigma_{l}^{2}}\right)
$$

where $m_{l}, \sigma_{l}$ are the mean (in decibels) and the variance of the $\log$-normal distribution, i.e., $c_{l}(d B)=20 \log \left(c_{l}\right)$. Since $c_{l}(t)$ is caused by terrain configuration and obstacles, the random process fluctuates over a much longer time scale. Again, from field test results, the order of time span for $c_{l}(t)$ is about $1 \mathrm{~s}$. Since mobile terminals are scattered geographically across the cell and are moving independently of each other, we assume the channel fading experienced by each mobile terminal is independent of each other.

2) Variable Throughput Channel Coding: Redundancy is incorporated to the information packet for error protection. To exploit the time-varying nature of the wireless channel, a variable rate channel-adaptive physical layer is employed as illustrated in Fig. 6. CSI $c(t)$, which is estimated ${ }^{1}$ at the receiver, is fed back to the transmitter via a low-capacity feedback channel. Based on the CSI, the level of redundancy and the modulation constellation applied to the information cells are adjusted accordingly by choosing a suitable transmission mode. ${ }^{2}$ Thus, the instantaneous throughput is varied according to the instantaneous channel state. In our study, a six-mode variable rate adaptive bit-interleaved trellis coded modulation scheme (ABICM) is employed [11]. Transmission

${ }^{1}$ In this paper, we assumed CSI is estimated by the pilot-symbol approach [11].

${ }^{2}$ Transmission mode refers to the combination of channel encoding rate and modulation constellation level. modes with normalized throughput ${ }^{3}$ varying from 1/2 to 5 are available depending on the channel condition. For real-time sources such as CBR or VBR, the physical layer employs a variable throughput forward error correction (FEC) code. For a nonreal-time source such as ABR, the physical layer employs a variable throughput error correction code embedded with error detection and retransmission.

Information cells per user are transmitted in the assigned traffic slots of a TDMA frame. Since the coherence time of short-term fading is around $20 \mathrm{~ms}$, the CSI remains approximately constant within a traffic slot duration. Hence, all the transmitted symbols of the traffic slot (per user) share the same transmission mode, which is determined by the current CSI level. Specifically, transmission mode $q$ is chosen if the feedback CSI, $\hat{c}$, falls within the adaptation thresholds, $\left(\zeta_{q-1}, \zeta_{q}\right)$. Here, the operation and the performance of the ABICM scheme is determined by the set of adaptation thresholds $\left\{\zeta_{0}, \zeta_{1}, \ldots\right\}$. Furthermore, we operate the ABICM scheme in the constant BER mode [11]. That is, the adaptation thresholds are set optimally to maintain a target transmission error level over a range of CSI values. When the channel condition is good, a higher mode could be used and the system enjoys a higher throughput. On the other hand, when the channel condition is bad, a lower mode is used to maintain the target error level at the expense of a lower transmission throughput. Note that when the channel state is very bad, the adaptation range of the ABICM scheme can be exceeded, making it impossible to maintain the targeted BER level. The concept of constant BER operation is illustrated in Fig. 7(a).

Given the above considerations about the channel state, the instantaneous throughput offered to the MAC layer $\rho$ is also variable and is, therefore, a function of the CSI $c(t)$ and the

${ }^{3}$ Normalized throughput refers to the number of information bits carried per modulation symbol. 


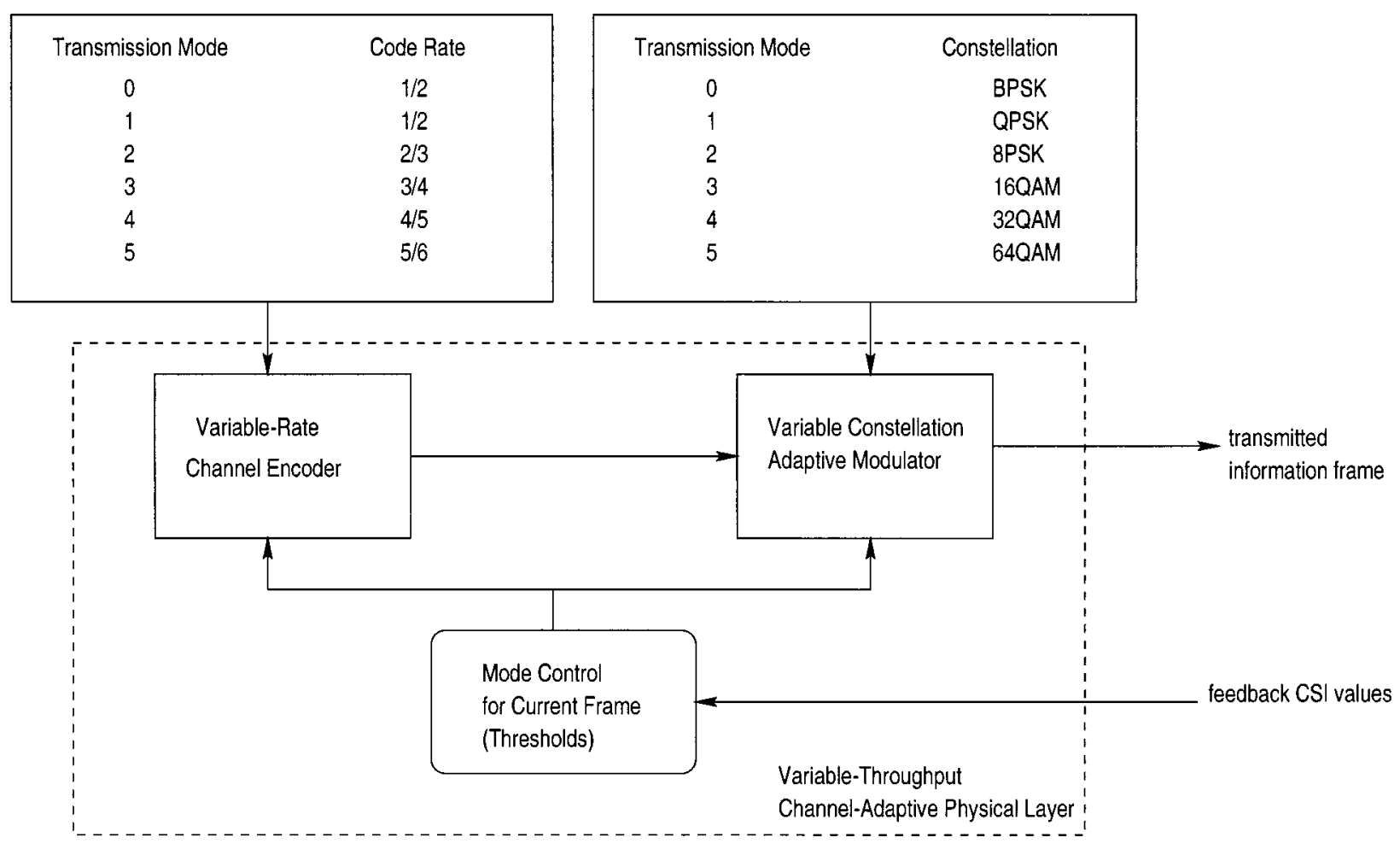

Fig. 6. A conceptual block diagram of the variable throughput channel adaptive physical layer.

target BER $P_{b}$ denoted by $\rho=f_{\rho}\left(c(t), P_{b}\right)$. Fig. 7(b) illustrates the variation of $\rho$ with respect to the CSI.

As indicated earlier, most of the previous schemes, bandwidth allocation in the MAC layer for a certain traffic type is essentially based only on a first-come-first-serve strategy. Some of the previous MAC schemes even do not consider CBR and VBR separately. For those MAC schemes that treat CBR, VBR, and ABR separately, no mechanism is incorporated to adjust the priority between classes in a flexible way. Most importantly, these protocols do not take into account of the CSI in the bandwidth allocation process. However, in a wireless communication system where burst errors due to fading are inevitable, CSI is a critical factor in achieving a higher overall utilization of the precious bandwidth in the system. This motivates the SCAMA protocol, which works closely and synergistically with the underlying channel adaptive physical layer. Specifically, the protocol adaptively assigns information slots to users based on their CSI ranking. Furthermore, the SCAMA protocol could accommodate CBR, VBR, and ABR traffic effectively with a very flexible priority adjustment between different classes.

3) Frame Structure: Fig. 8 shows the uplink and the downlink frame structure of the SCAMA MAC protocol. To incorporate the channel-adaptive feature of the SCAMA MAC protocol, the TDMA frames for the uplink and downlink are divided into subframes as follows.

In the uplink, a frame is divided into three subframes as illustrated in Fig. 8(a). They are the request subframe, the traffic subframe, and the reporting subframe. Specifically, there are $N_{r}$ mini slots in the request subframe for CBR, VBR, and ABR requests requests contention. Note that an $\mathrm{ABR}$ user is not allowed to make reservation in the sense that even if an $A B R$ user is granted traffic slot(s) in the current frame, it has to contend again in the next frame for the remaining data cells. On the other hand, CBR and VBR users can reserve slots in succeeding frames. Specifically, when a CBR or VBR user successfully makes a transmission request in one of the $N_{r}$ mini slots, the user does not need to contend again in the next period and the request will be automatically generated in the MAC layer until the current burst ends. There are $N_{i}$ information slots in the traffic subframe for the transmission of CBR, VBR, or ABR packets. Finally, there are $N_{b}$ mini slots in the reporting subframe. The functions of the three subframes will be elaborated in detail later in Section III-G4. The frame duration is $2.5 \mathrm{~ms}$. Such a short frame duration has the advantage of shorter delay and is practicable in wideband systems [17].

A downlink FDD frame is similarly partitioned into four subframes, namely the acknowledgment subframe, the polling subframe, the traffic subframe, and the announcement subframe. The frame duration is also $2.5 \mathrm{~ms}$ and the number of slots in the subframes are given by $N_{r}, N_{b}, N_{i}$, and $N_{b}$, respectively. The functionality and operation of each subframe are described in Section III-G4. Note that in both uplink and downlink, variable throughput adaptive channel coding and modulation is applied to traffic slots only. For the mini slots of the other subframes, traditional QPSK modulation is applied.

4) Protocol Operations: The operation of the SCAMA protocol is divided into two phases, namely the request phase and transmission phase. In the request phase, mobile terminals which have packets to transmit will send a request packet in one of the $N_{r}$ request mini slots, governed by the respective permission probability. The request packet is short (24 bits), ${ }^{4}$

\footnotetext{
${ }^{4}$ The 24-bit packet size includes the payload only. Other common header and trailer bits, such as guard bits and CRC bits are not shown here for brevity.
} 


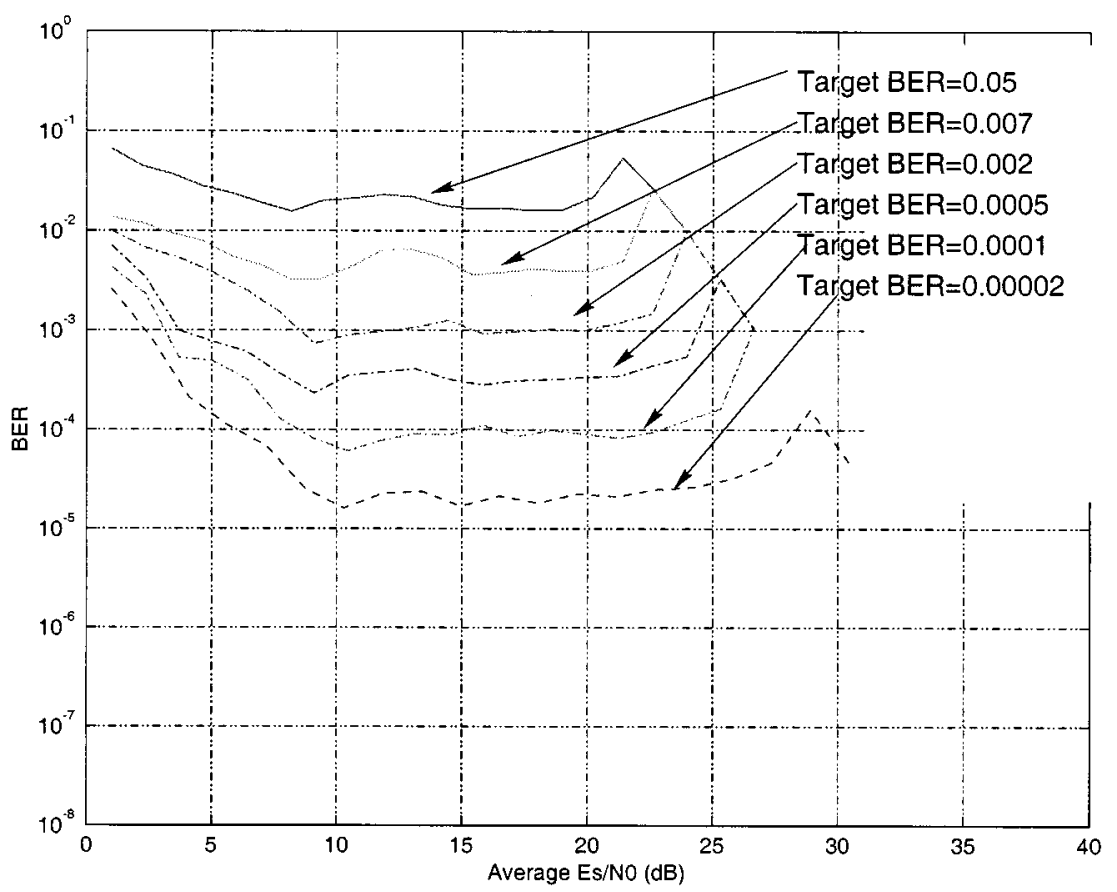

(a)

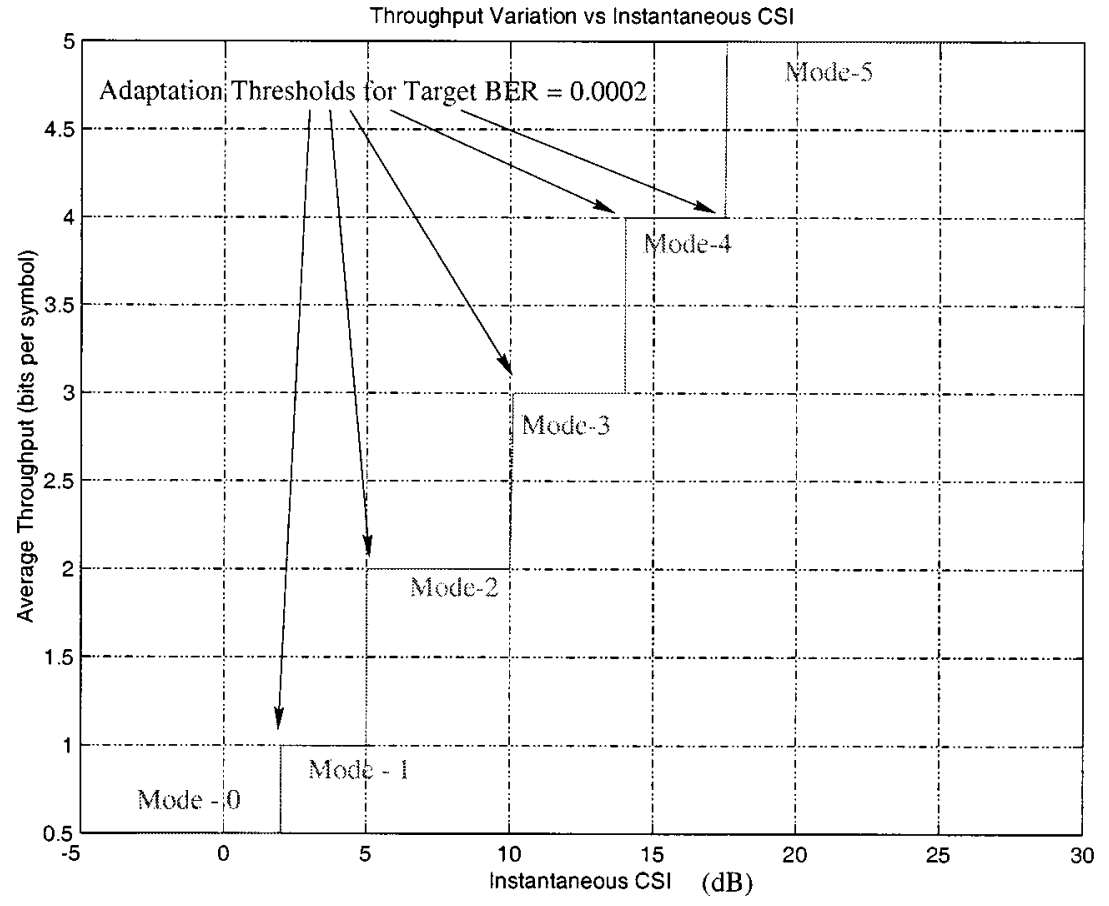

(b)

Fig. 7. BER and throughput of ABICM scheme.

occupying only a mini slot, as illustrated in Fig. 8(a). It contains the mobile terminal identity (ID), request type (CBR, VBR, or ABR), data deadline, number of information data cells desired to transmit as well as pilot symbols for CSI estimation. If more than one mobile terminals send request packets in the same request mini slot, collision occurs and all the request packets are lost if capture effect is not considered (if capture is considered, the request with the highest signal energy may be successfully received). After each request mini slot, an acknowledgment packet will be broadcast from the base station through the acknowledgment mini slot in the downlink frame as illustrated in Fig. 9(a). The acknowledgment packet contains only the successful request packet ID. Mobile terminals that fail to receive an acknowledgment will retransmit the request packet in the next request mini slot, again governed by the permission probability. On the other hand, successfully acknowledged users will wait for announcement on the allocation schedule of the traffic slots from the base station.

Unlike traditional MAC protocols, the base station will collect all requests in the current request phase as well as the 


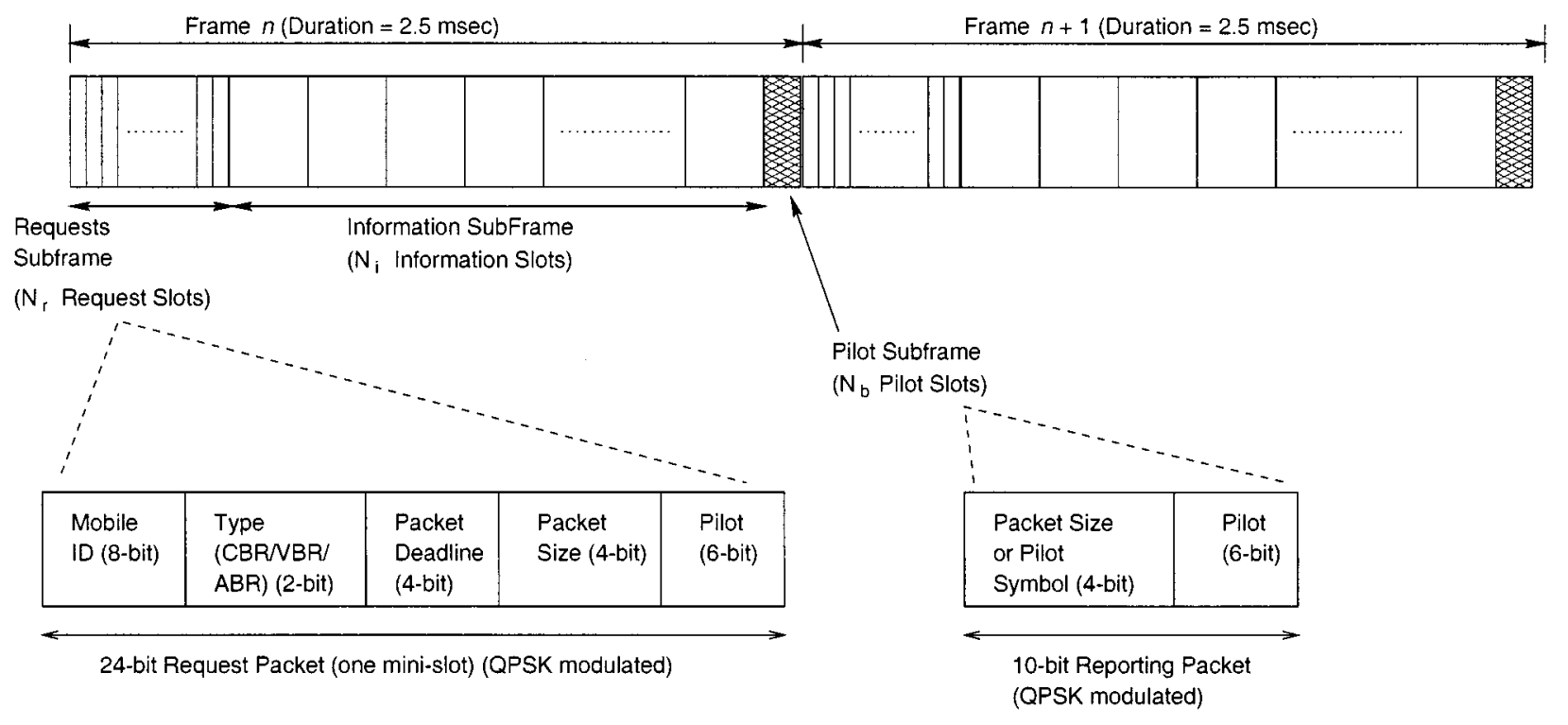

(a)

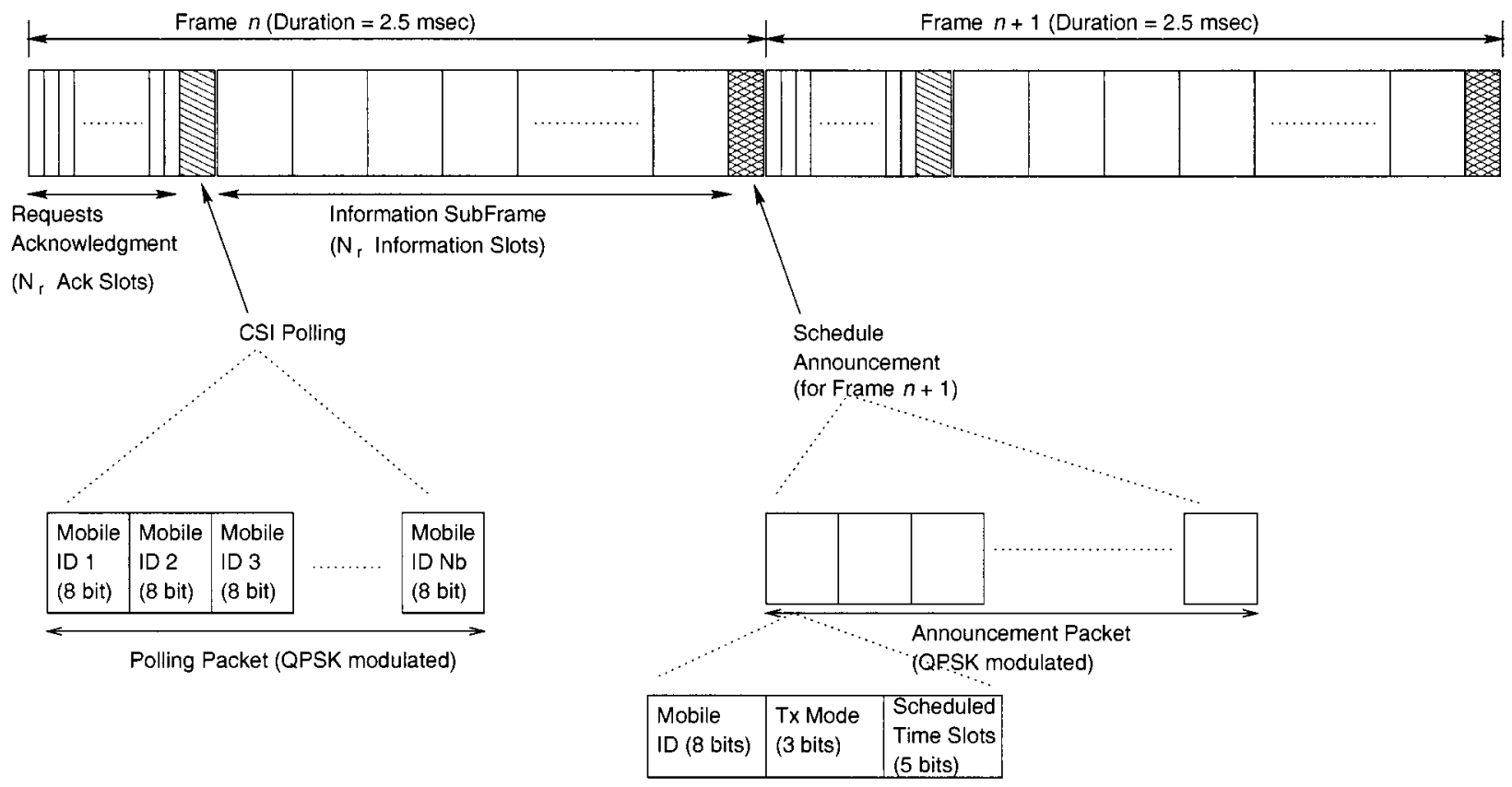

(b)

Fig. 8. Frame structures of the SCAMA protocol for CBR, VBR, and ABR users.

backlog requests from the previous frames before allocation of traffic slots. All the requests will be assigned priorities according to the deadline, CSI, service type (CBR, VBR, or $\mathrm{ABR}$ ), as well as the waiting time of the request (i.e., the number of elapsed frames since the request is acknowledged). The time-slot allocation algorithm is conceptually depicted in Fig. 9(b). Since the physical layer offers a variable throughput which is dependent on the CSI, the rationale behind the SCAMA MAC protocol is to give higher priority to the mobile terminals that are in better channel condition in the bandwidth allocation process. The motivation of this strategy is that a user with better channel condition, with the support of the variable rate channel encoder, can enjoy a larger throughput and, therefore, can use the system bandwidth more effectively.
Nevertheless, for fairness's sake, information slots should also be allocated to mobile terminals that are approaching their deadlines, despite their possibly worse channel states; otherwise, the queued information packets will be dropped.

5) Priority Function for Slots Allocation: In general, a priority function for efficient slots allocation should satisfy the following objectives:

- give priority to requests with high CSI value;

- maintain priority (i.e., prevent priority inversion) between different classes (CBR, VBR, or ABR);

- maintain fairness (delay jitter) within each class.

In the SCAMA protocol, we employ a general priority function which could provide a flexible balance of the above conflicting goals. Furthermore, the slots allocation mechanism is 
also very flexible for incorporating other types of allocation algorithms such as deficit round robin, weighted fair queueing, and class-based queueing [19].

Specifically, the priority metric of the $i$ th request (which may be a new request or a backlog request), $\mu_{i}$, is given by (2), shown at the bottom of the page, where $T_{d}^{(i)}, T_{w}^{(i)}, \lambda_{\mathrm{CBR}}, \lambda_{\mathrm{VBR}}, \lambda_{\mathrm{ABR}}$, $\beta_{\mathrm{CBR}}, \beta_{\mathrm{VBR}}, \beta_{\mathrm{ABR}}, \Delta_{\mathrm{CBR}}$, and $\Delta_{\mathrm{VBR}}$ are the deadline, the waiting time, the forgetting factors of the CBR, VBR, and ABR requests, as well as the priority offsets assigned to the CBR, VBR, or ABR users, respectively. From (2), the first term is aimed to enforce that a higher priority for requests with a higher throughput. The second term is to maintain fairness ${ }^{5}$ within each of the service classes. Finally, the last term is responsible for maintaining priority between different classes. As will be demonstrated in Section IV, the balance between the three goals could be easily adjusted by tuning $\lambda$ and $\Delta$.

Thus, in the allocation phase, traffic slots are allocated to service requests according to the sorted priority metrics. If there are not sufficient traffic slots to service all requests, remaining requests are queued and reconsidered in the next frame. ${ }^{6}$ After the request phase, the results of traffic slot allocation will be broadcast in the announcement subframe of the downlink frame. The announcement packet contains the traffic slot allocation schedule as well as the transmission mode as illustrated in Fig. 8(b). Mobile terminals will then start to transmit information packets on the allocated traffic slot(s).

6) Handling Heterogeneous Users Requirements: The SCAMA protocol is reservation based for CBR and VBR users only. As mentioned earlier, for a ABR user, even if traffic slots have been assigned for its successfully acknowledged request, the allocation is meant only for the current frame and the $\mathrm{ABR}$ user has to initiate another request for any remaining data cells. By contrast, for a CBR or VBR user, when traffic slots have been assigned for its successfully acknowledged request, additional requests will be automatically generated by the base station (hence, reservation) periodically at $20 \mathrm{~ms}$ (i.e., taking voice as an example CBR source) and $40 \mathrm{~ms}$ (i.e., taking video as an example VBR source) time intervals for CBR and VBR, respectively. Thus, the CBR or VBR user does not need to contend for request mini slots anymore in the current talkspurt. By avoiding unnecessary requests, the advantage of this reservation strategy is the reduction of the contention collisions in the request phase. For a CBR user, the number of cells generated per CBR period is constant and, hence, this basic reservation scheme works fine. However, for a VBR user, the number of cells generated per VBR period is a random variable and hence, this information needs to be updated per

\footnotetext{
${ }^{5}$ The second term will be large for requests with a urgent deadline or long waiting time.

${ }^{6}$ If the deadline for a remaining request has expired, this request will not be queued anymore. The information packet at the mobile terminal will be dropped.
}
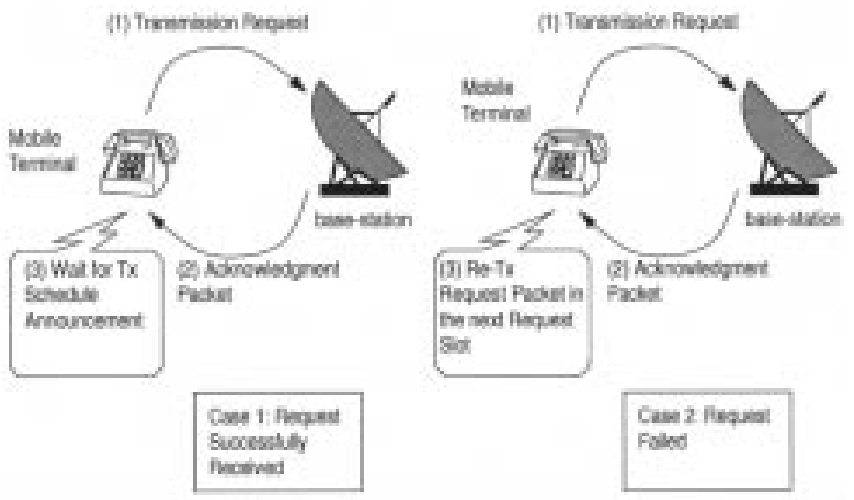

(a)

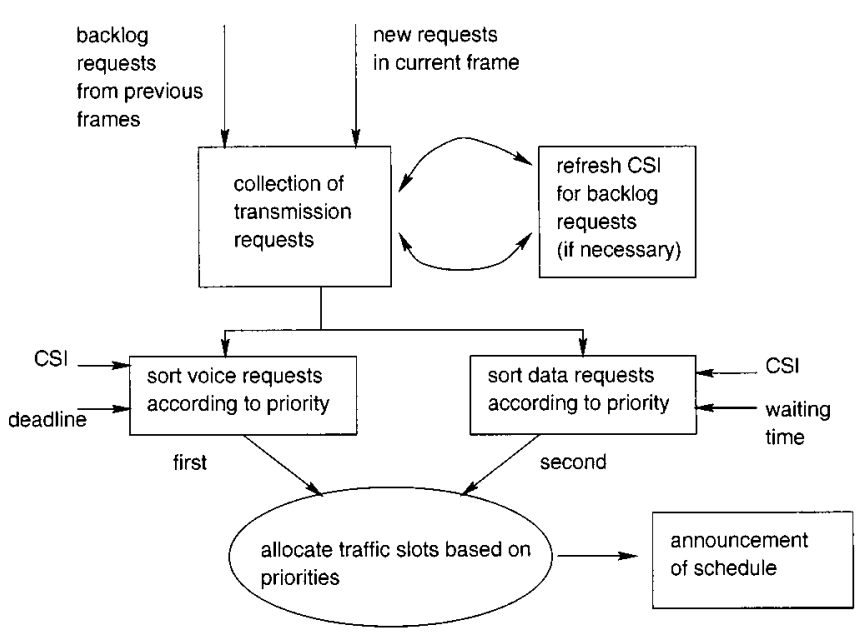

(b)

Fig. 9. Operations of the channel-adaptive MAC protocol (SCAMA) for wireless ATM.

VBR period in order to make proper reservation for VBR. The mechanism for VBR updating is illustrated in the following section.

7) CSI Determination: On the other hand, a critical component in the SCAMA MAC protocol is the determination of the current CSI for each user. As mentioned earlier, we assume that the coherence time for short-term fading is around $20 \mathrm{~ms}$, while the frame duration is only $2.5 \mathrm{~ms}$. Thus, the CSI remains approximately constant for at least two frames. For a new request, known pilot symbols are embedded in the request packets so that the current CSI can be estimated at the base station and this estimated CSI is valid for the next few frame duration. However, for a backlog request, the estimated CSI value obtained previously during a past request phase may be obsolete and thus, a mechanism is needed to obtain update the CSI.

Both the VBR reservation and the CSI update relies on a special updating procedure, which is illustrated in Fig. 10.

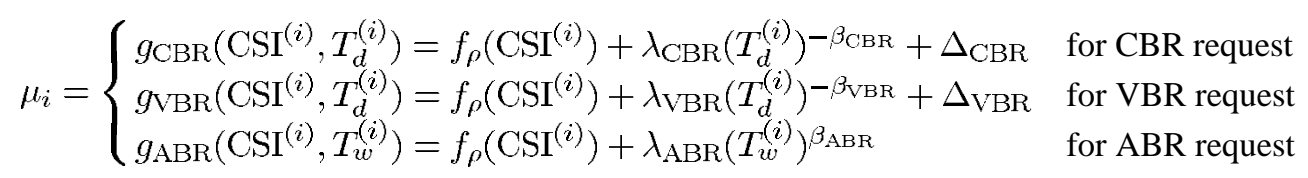




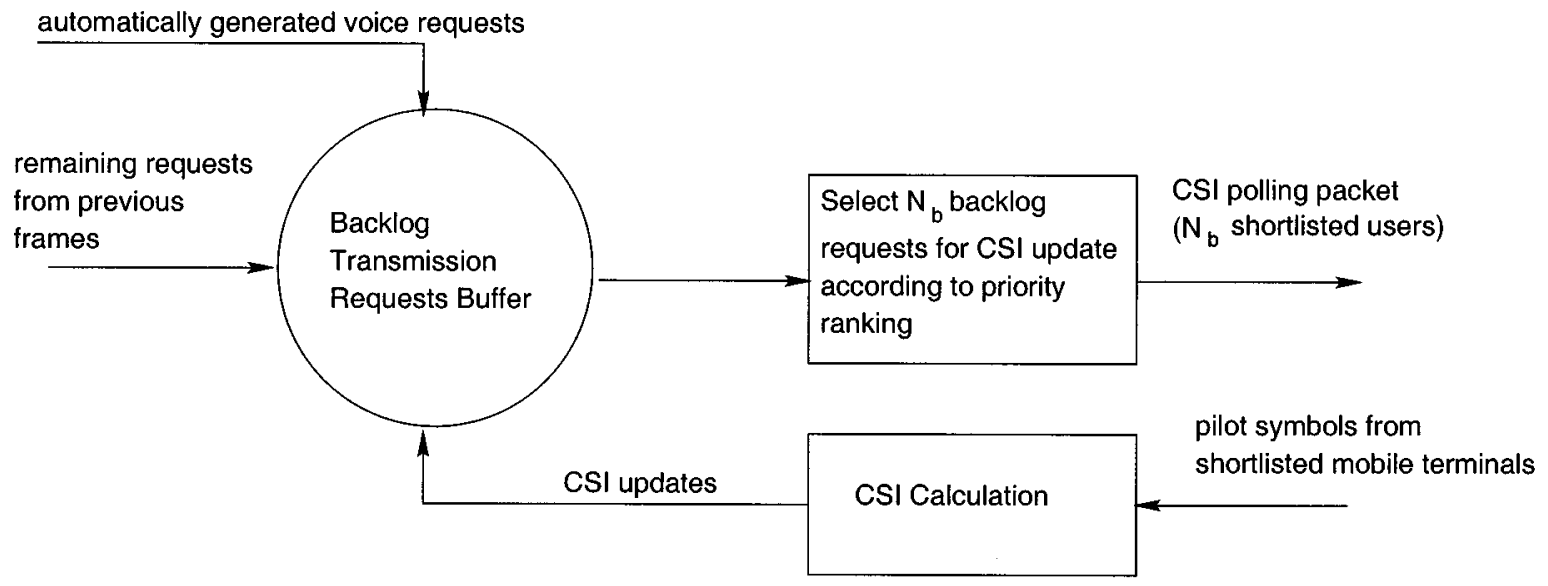

Fig. 10. Information (CSI or VBR demands) updating mechanism for backlog requests.

At the beginning of each frame, the base station short lists $N_{b}$ backlog requests (those with the CSI values expired or those requiring VBR reservation update) according to their priorities. A polling packet is then broadcast to the mobile terminals in the polling subframe. The CSI polling packet contains the mobile terminal IDs that are short listed by the base station. The structure of the polling packet is shown in Fig. 8(b). Mobile terminals listed in the polling packet respond at the appropriate reporting mini slot according to the order specified in the polling packet. If the short listed request is of CBR type with CSI value expired, the mobile station will transmit pilot symbols in the reporting mini slot. Otherwise, the mobile station will transmit a VBR demand update packet as shown in Fig. 8(a). The VBR demand update packet contains the number of VBR cells generated in the current frame as well as pilot symbols for CSI updating.

Thus, the base station could update the VBR reservation requests as well as backlog requests' CSI values (which are valid for at least two consecutive frames). The estimated CSI value is used to determine the transmission mode in the physical layer as well as to determine the priority of the request in the MAC layer. With the above considerations, the SCAMA protocol is outlined below in pseudocode format.

\section{1 loop}

2 contention phase \{requests are acknowledged but no slot is assigned $\}$

$3 \quad\{$ CSI of each request is recorded

4 merge the new requests to the request queue

5 sort the request queue according to the request priority

6 assign information slots according to the request queue order

7 remaining unassigned requests are queued

8 end loop

\section{RESULTS AND INTERPRETATIONS}

In this section, we present the performance results of our performance comparison of the seven protocols. In our simulation study, we assume a transmission bandwidth of $1.36 \mathrm{MHz}$ for the TDMA frames. CBR bit rate is $8 \mathrm{~kb} / \mathrm{s}$ while the average VBR bit rate is $128 \mathrm{~kb} / \mathrm{s}$. Mobile users are assumed to be moving in
TABLE II SIMULATION PARAMETERS

\begin{tabular}{ll}
\hline Parameter & Value \\
\hline$N_{T}$ & 10 \\
$N_{i}$ & 40 \\
$N_{b}$ & 20 \\
$t_{t}$ & $1000 \mathrm{msec}$ \\
$t_{s}$ & $1350 \mathrm{msec}$ \\
$p_{c}$ & 0.3 \\
$p_{v}$ & 0.3 \\
$p_{a}$ & 0.2 \\
channel bandwidth & $1.36 \mathrm{MHz}$ \\
CBR (voice) data rate & $8 \mathrm{kbps}$ \\
VBR (video) data rate & $128 \mathrm{kpbs}$ \\
ABR data rate & $16 \mathrm{kbps}$ \\
number of simulated frames & $2 \times 10^{6}$ \\
\hline
\end{tabular}

a random way at a maximum speed of $40 \mathrm{~km} / \mathrm{h}$ in a microcell environment. Table II summarizes the parameters we used.

\section{A. Source Models}

The wireless ATM system considered in this paper is aimed to support integrated $\mathrm{CBR}, \mathrm{VBR}$, and ABR services. As such, we assume that there are only three types of mobile terminals, namely the CBR terminal, the VBR terminal and the ABR terminal in the system. Both CBR and VBR cells are assumed to be delay sensitive while ABR cells are assumed to be delay insensitive. Thus, CBR and VBR cells are labeled with deadlines. The cells will be dropped by a mobile terminal if the deadline expires before being transmitted. Such cells dropping has to be controlled to within a certain limit (e.g., below $1 \%$ for voice as indicated in [6]) in order to have acceptable quality of service for CBR and VBR users. The source and contention models are summarized as follows.

- CBR Source Model: We use voice as an example CBR source. The voice source is assumed to be continuously toggling between the talkspurt and silence states. The duration of a talkspurt and a silence period are assumed to be exponentially distributed with means $t_{t}$ and $t_{s}$ seconds, respectively (as indicated by the empirical study in [14], $t_{t}=1$, and $t_{s}=1.35$ ). We assume a talkspurt and a 


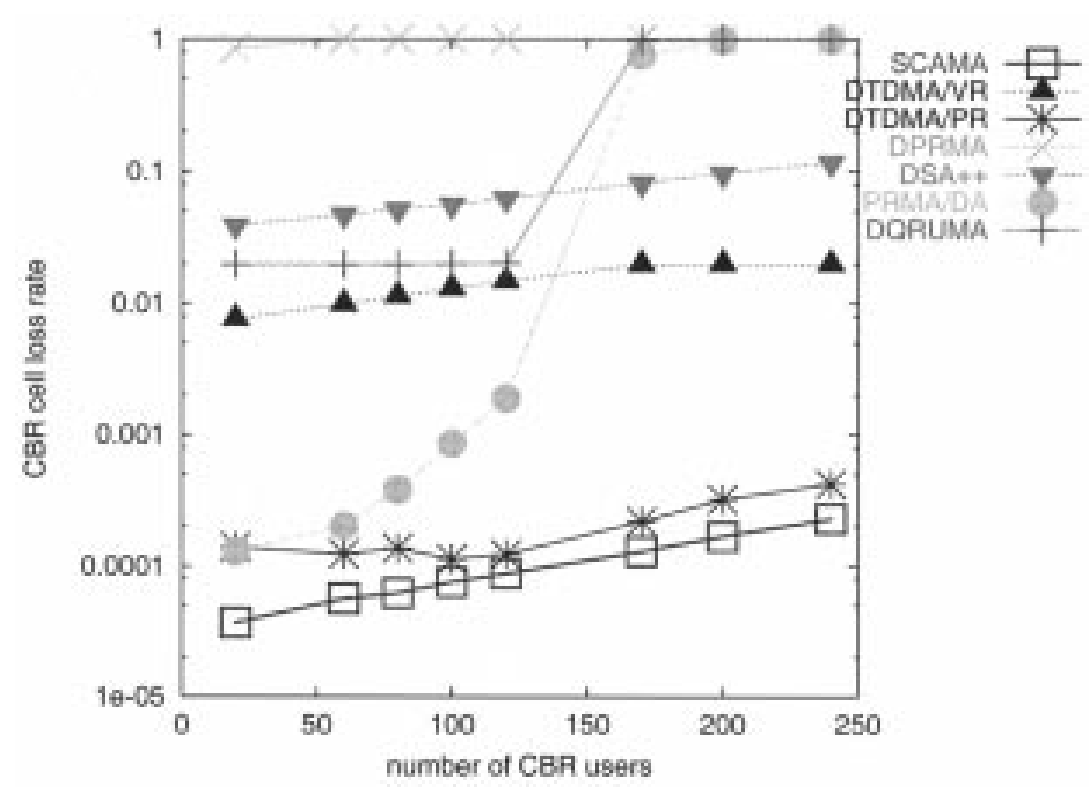

(a)

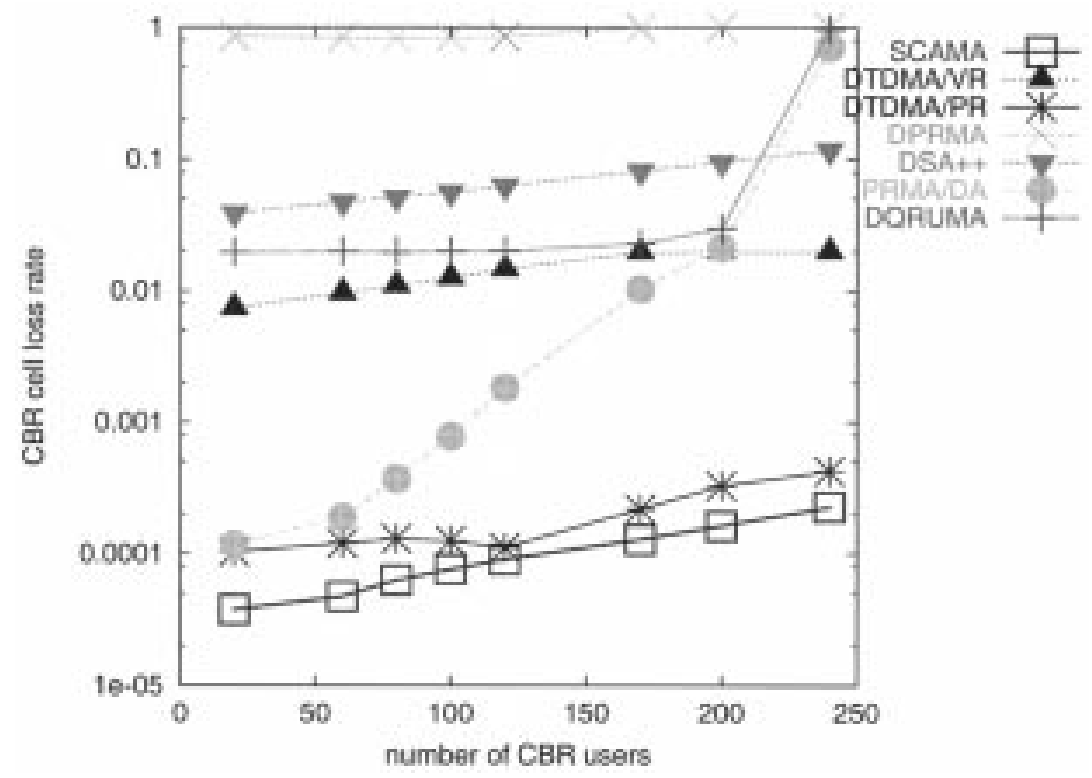

(b)

Fig. 11. Performance of the protocols for CBR users (20 VBR users).

silence period start only at a frame boundary. Finally, as mentioned above, a voice source cannot tolerate a cell-loss rate higher than $1 \%$ in order to achieve a reasonable service quality [6].

- VBR Source Model: We use video teleconference as an example VBR source. In the model we use [10], the number of cells per VBR period (i.e., $40 \mathrm{~ms}$ for a 25 fps frame rate) is govern by the DAR(1) model, which is a Markov chain characterized by three parameters: the mean, the variance, and $\rho$. The transition matrix is computed as

$$
P=\rho I+(1-\rho) Q
$$

where $\rho$ is the autocorrelation coefficient and $I$ is the identity matrix. Furthermore, each row of $Q$ is identical and consists of the negative binomial probabilities $\left(f_{0}, \ldots, f_{K}, F_{K}\right)$, where $F_{K}=\Sigma_{k<K} f_{k}$, and $K$ is the peak rate. Similar to a voice source, a video source can only tolerate a $1 \%$ cell-loss rate [10].

- ABR Source Model: The arrival time of data generated by a ABR data terminal is assumed to be exponentially distributed with mean equal to one second. The data size, in terms of number of cells, is also assumed to be exponentially distributed with mean equal to 100 cells. An ABR user will not drop cells because there is no deadline constraint. Again we assume that the cells arrive at a frame boundary.

- Terminal Contention Model: As in most previous studies, to avoid excessive collisions, even if a user has some cells awaiting to be sent, the user will attempt to send a request at a request mini slot only with a certain permission probability. The permission probability for $\mathrm{CBR}, \mathrm{VBR}$, and ABR users are denoted by $p_{c}, p_{v}$ and $p_{a}$, 


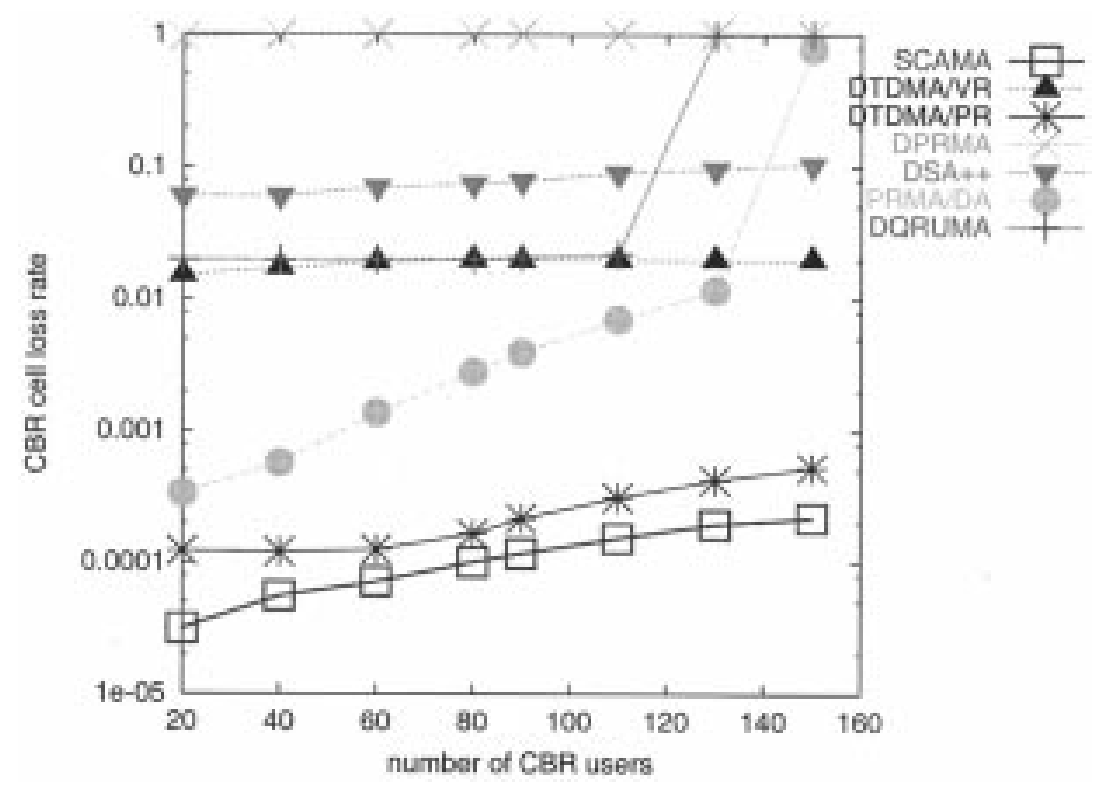

(a)

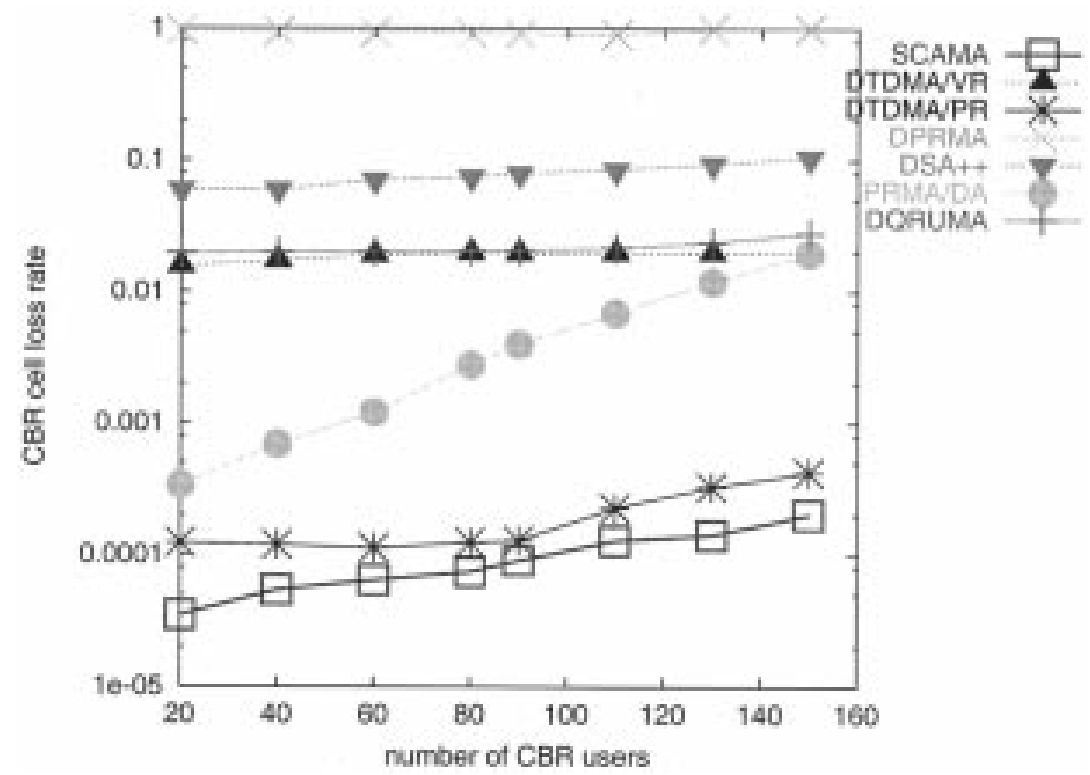

(b)

Fig. 12. Performance of the protocols for CBR users (25 VBR users).

respectively. The protocol also has a request queue which stores the previous requests that survive the contention but are not allocated information slots.

It is also interesting to investigate the performance of the seven protocols under situations with and without capture. Thus, the capture process is also implemented. Specifically, suppose there are $k$ requests, with signal power denoted by $P_{1}, P_{2}, \ldots, P_{k}$ contending for a request mini slot. A request $j$ can be captured if [1]

$$
\frac{P_{j}}{\sum_{i \neq j} P_{i}}>\gamma
$$

where $\gamma$ is the SNR threshold.

1) CBR Performance: The service quality for CBR users, being voice sources, is governed by the average cell-loss rate,
$P_{\text {loss }}$, which is contributed by two factors: cell dropping at the mobile and cell loss during transmission. On one hand, voice cell is delay sensitive and, hence, voice cells are labeled with deadlines. A voice cell has to be discarded if its delay exceeds the deadline. ${ }^{7}$ Such discarding constitutes the cell dropping at the voice terminal. On the other hand, transmitted cells could be corrupted due to channel error and, thus, cell transmission error results. The cell-loss rate $P_{\text {loss }}$ is then given by

$$
P_{\text {loss }}=\frac{N_{t x}-N_{r v}}{N_{t x}}
$$

where $N_{t x}$ and $N_{r v}$ are the number of transmitted voice cells and the number of voice cells received without error, respectively.

${ }^{7}$ In this paper, the deadline of voice cell is assumed to be $20 \mathrm{~ms}$ after it is generated by the source. 


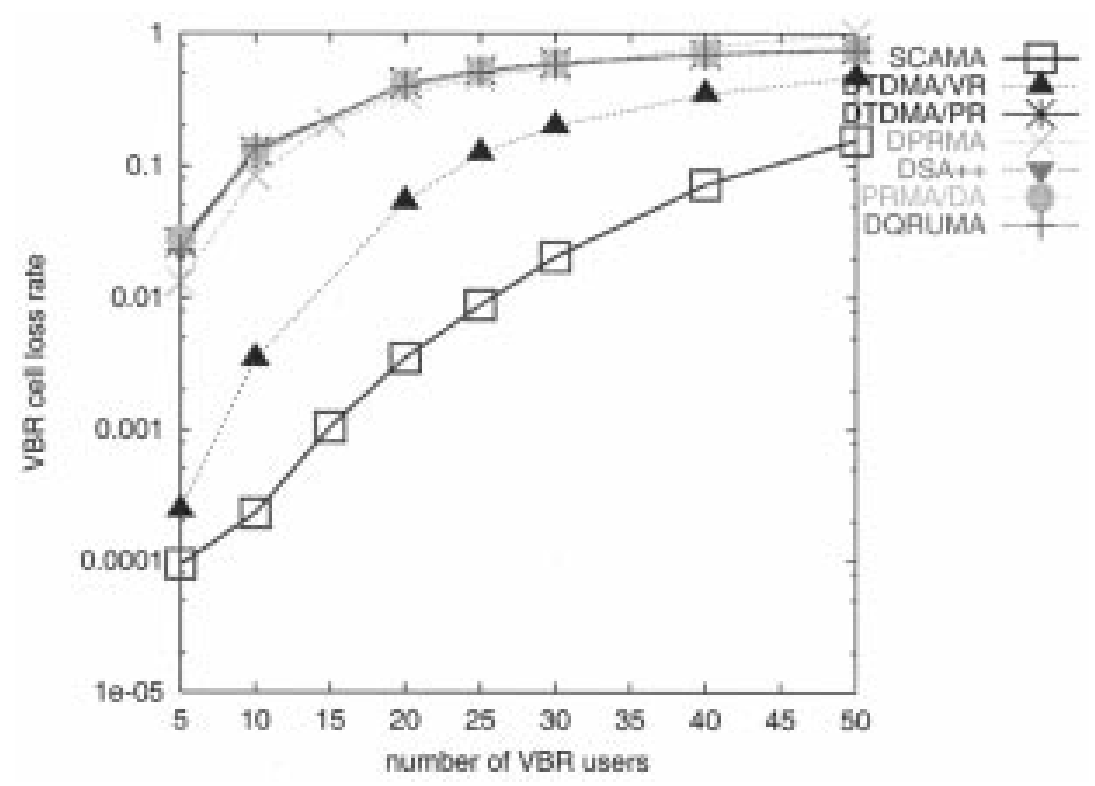

(a)

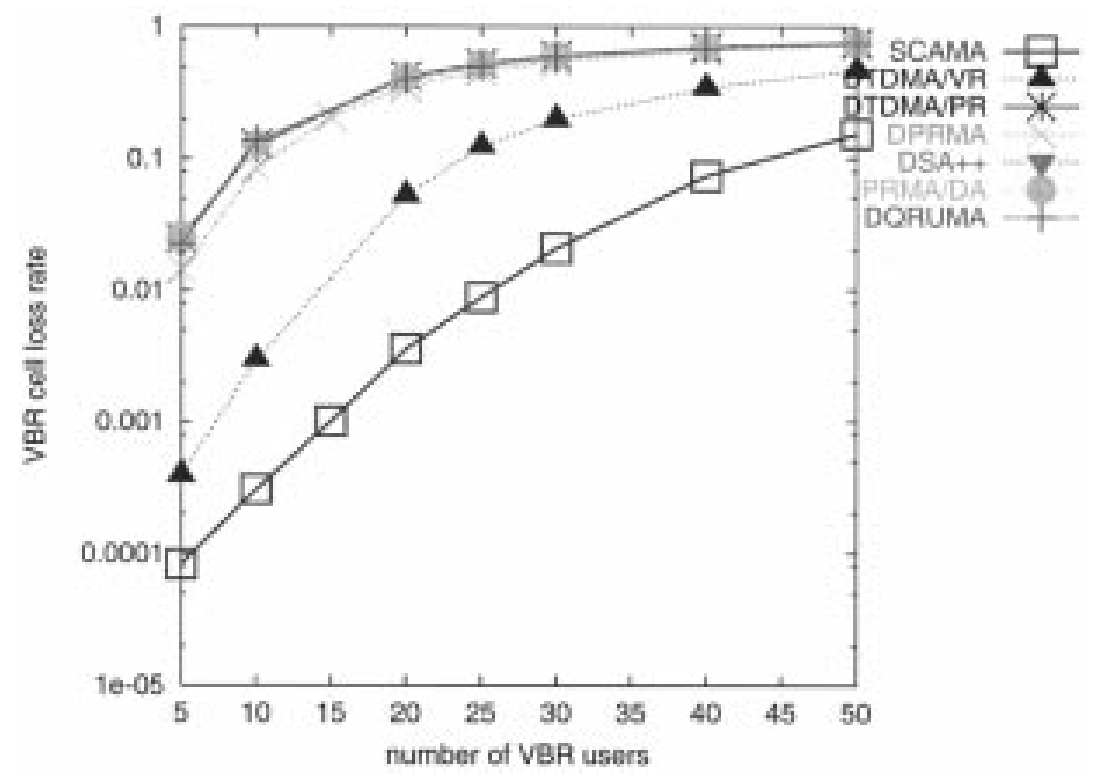

(b)

Fig. 13. Performance of the protocols for VBR users (30 CBR users).

Figs. 11 and 12 show the cell-loss rate performance of the seven protocols for the CBR users. In this set of experiments, we varied the number of $C B R$ users in the system, while we fixed the number of ABR users to be 25 and that of VBR users to be 20 and 25 also. Consider the results shown in Fig. 11(a). As can be seen, the DPRMA protocol performs much worse compared with the other protocols. The DPRMA protocol cannot even handle as few as ten CBR users. Indeed, the DPRMA protocol was found to perform well in the range of a few VBR users, as demonstrated in [4]. The DSA++, DQRUMA, and DTDMA/VR protocols in general perform better than the DPRMA protocol. However, the cell-loss rates achieved in these three protocols are still much higher than the $1 \%$ tolerance threshold for a CBR (voice) user. This can be explicated by the fact that the DQRUMA and DTDMA/VR protocols do not incorporate any prioritization mechanism in the time slots allocation process. That is, the time slots are essentially allocated in a first-come-first-serve manner. Thus, as VBR and ABR users usually have a much larger chunk of cells for transmission, the CBR users suffer from the "convoy" effect (i.e., a large request blocks a number of subsequent small requests) and, thus, cell droppings occur quite frequently. In addition, among these three protocols, the DQRUMA is the least stable. Indeed, when the number of CBR users reaches 150 , the protocol exhibits some thrashing effect-users keep contending with actually transmitting. On the other hand, the PRMA/DA, DTDMA/PR, and the SCAMA protocols perform much better than the former four protocols. The cell-loss rate is two order of magnitude lower. Note that these three protocols allocate slots according to the requests' priorities. In particular, isochronous users such as CBR and VBR users are given a higher priority than the ABR users. Thus, the results indicate that the priority functions used in these protocols are superior to those 


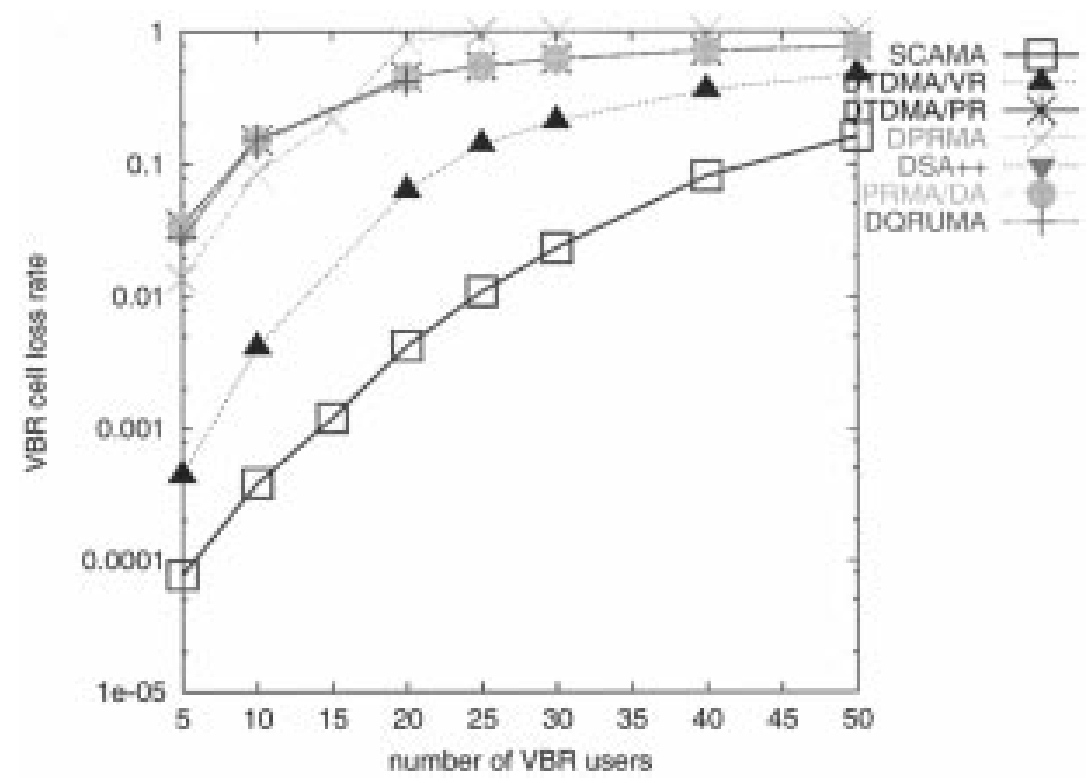

(a)

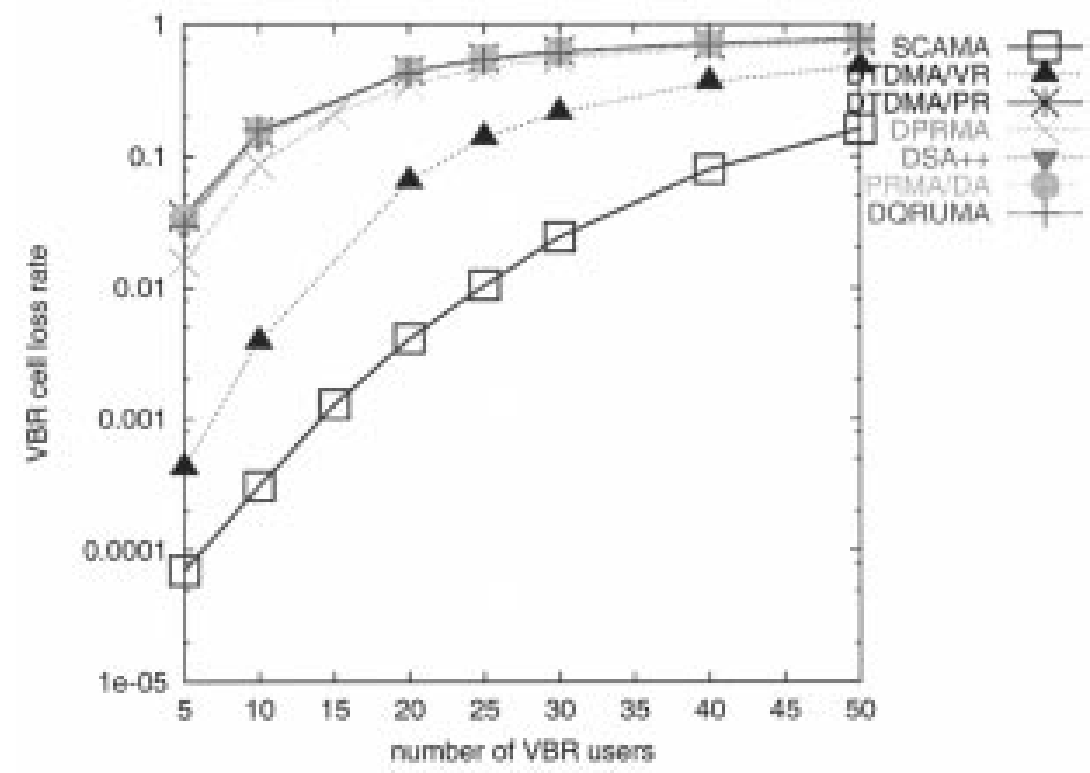

(b)

Fig. 14. Performance of the protocols for VBR users (50 CBR users).

used in the former four protocols. Furthermore, the channel adaptive protocol performs the best because the cell loss due to dropping can be avoided almost completely by the intelligent slots allocation algorithm. The ranking of the protocols for CBR users is: SCAMA, DTDMA/PR, PRMA/DA, DTDMA/VR, DQRUMA, DSA ++, DPRMA. Finally, we can see that for all seven protocols, including more VBR users in general slightly lowers the capacity of the system. The capture effect in general makes the protocols more stable even for high load.

2) VBR Performance: Similar to CBR users, the service quality of VBR users is also sensitive to loss (e.g., the image quality degrades if some cells are lost in a vidoe-conferencing application). Also, being a isochronous source, cells have deadlines such that missing the transmission deadlines render the cells useless. Thus, we also evaluate the performance of the protocols using the cell-loss rate for VBR users. Figs. 13 and 14 depict the VBR cell-loss rate performance of the seven protocols for cases with 25 ABR users and 30/50 CBR users. We can see that in contrast to CBR performance, the DTDMA/PR protocol performs quite poorly for VBR users in that the cell-loss rate is much higher than the $1 \%$ threshold even for five VBR users. This phenomenon is a result of assigning higher priority to CBR users in the DTDMA/PR, which concurs with the results presented in [14]. The DQRUMA, DPRMA, DSA ++ , and PRMA/DA protocol also gives similar performance as the DTDMA/PR protocol. The DTDMA/VR protocol, however, outperforms these five protocols by a large margin for VBR users. This is because the variable-throughput physical layer offers almost two times the average throughput compared with traditional fixed-throughput physical layers and is reflected in the performance of DTDMA/VR. Finally, we can see that the SCAMA protocol considerably outperforms the 


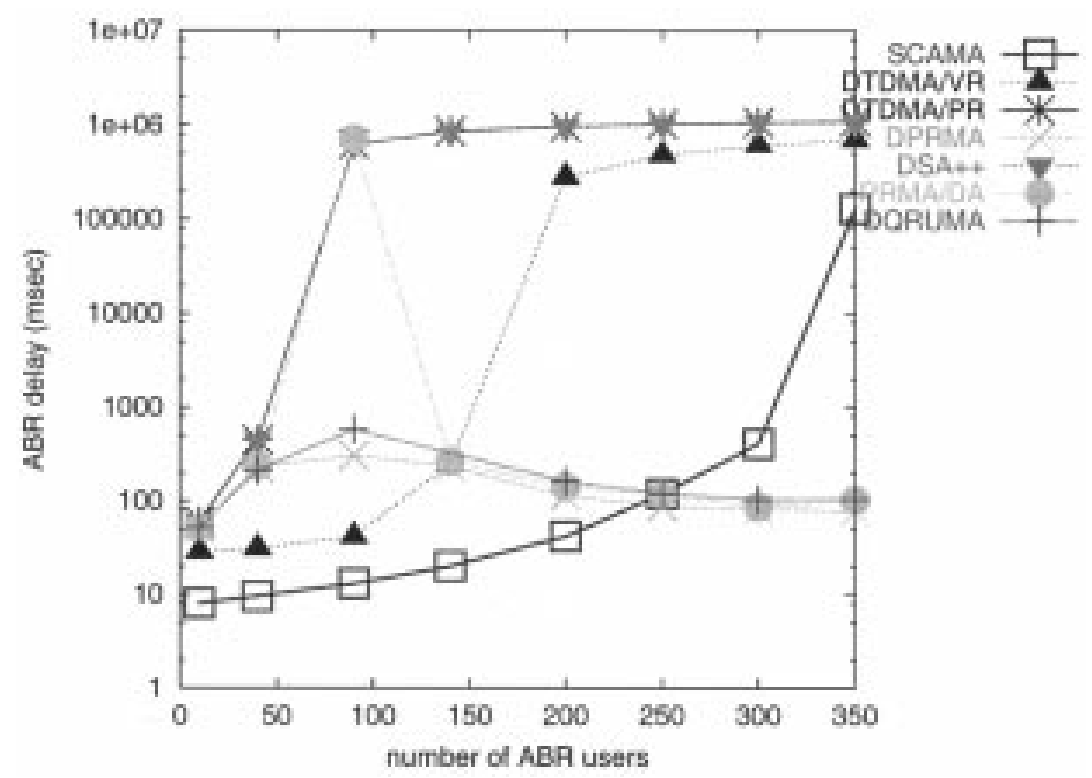

(a)

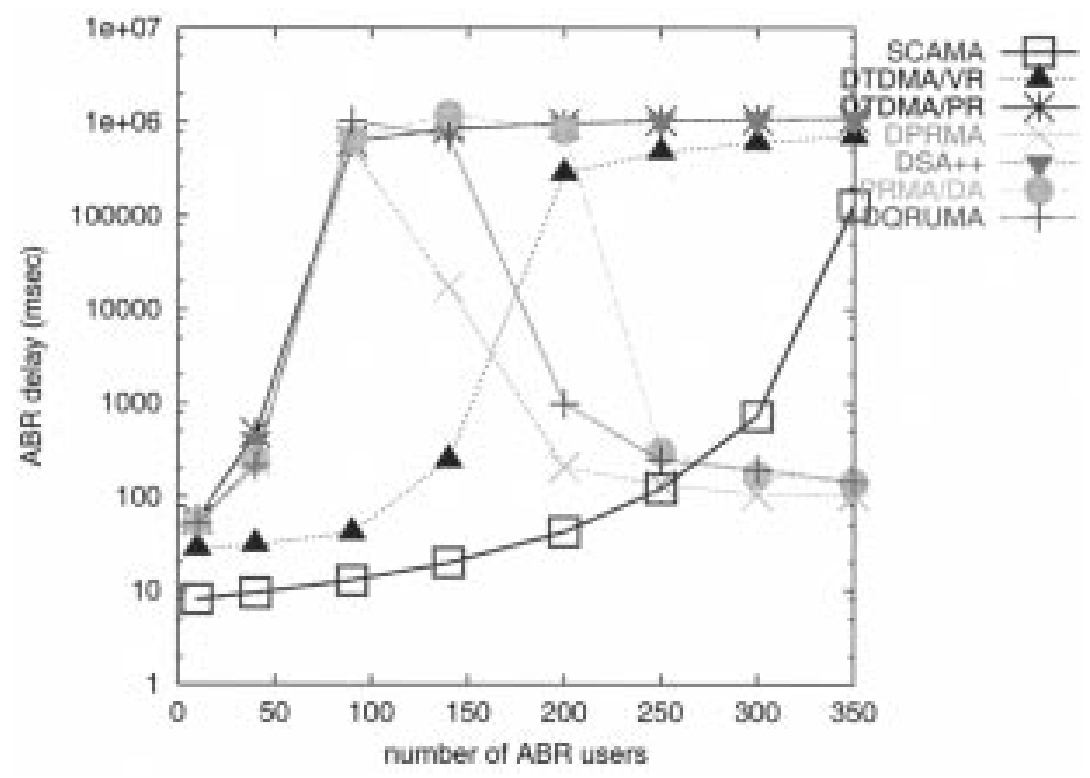

(b)

Fig. 15. Performance of the protocols for ABR users (delay versus traffic load; five VBR users).

DTDMA/VR protocol. This illustrates the synergy that could be achieved by the judicious requests prioritization (based on CSI, urgency, and throughput) process in the former. Also, in contrast to the CBR performance, the capture effect only slightly improves the performance of protocols. The remaining VBR results show a similar trend.

3) ABR Performance: ABR cells are delay insensitive and as such, they will not be discarded at the mobiles. However, ABR cells may experience transmission errors when the channel condition is poor. Thus, lost cells are retransmitted (through the data-link layer). This inevitably introduces additional delay due to retransmissions. Here, different from CBR and VBR users, ABR users' performance is quantified by two measures: delay and throughput. The average ABR data throughput $\bar{\rho}$ is defined as the average number of cells successfully received at the base station per frame. The average delay $\bar{D}_{d}$ is defined as the average time that a cell spends waiting in the transmitter buffer until the beginning of the successful transmission. Figs. 15-18 illustrate the performance of data terminal in terms of $\bar{D}_{d}$ and $\bar{\rho}$, respectively, for cases with $5 / 10 \mathrm{VBR}$ users and $20 \mathrm{CBR}$ users. When the traffic load is high, the system is in a highly congested state so that the average per-user throughput drops and the average per-user delay also increases dramatically. These adverse phenomena are detrimental to the data users' QoS, which depends critically on the parameters pair (delay, throughput) as describe above. Before the system gets into the congested state, the SCAMA protocol consistently offers a much lower delay and a much higher throughput compared to the other three protocols. In other words, given a certain QoS level, the SCAMA protocol can support a much larger ABR user population. For example, at a QoS level of (1 sec, 0.25), the SCAMA protocol can support more than 300 ABR users 


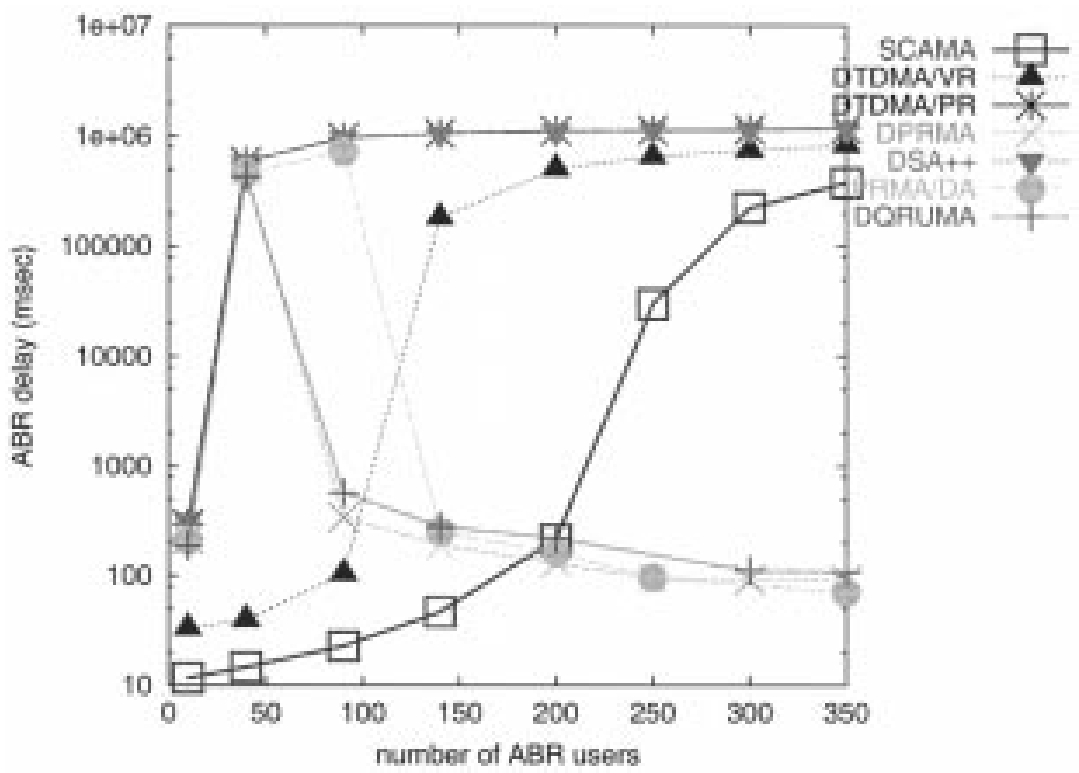

(a)

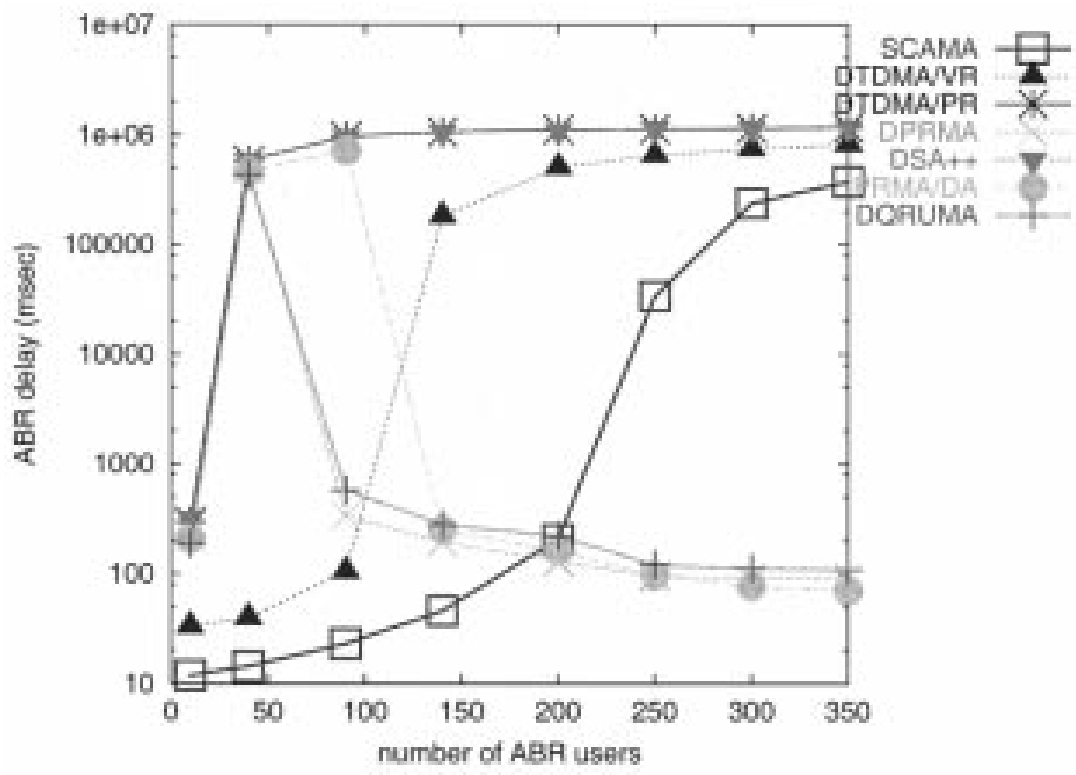

(b)

Fig. 16. Performance of the protocols for ABR users (delay versus traffic load; ten VBR users).

[see Fig. 15(a)] while the second best protocol, the DTDMA/VR protocol, can only support $100 \mathrm{ABR}$ users. Finally, as in the case of VBR, the capture effect does not help much. The other results give similar observations.

\section{B. Interpretations}

From the simulation results shown above, the SCAMA protocol is robust and outperforms six recently proposed efficient protocols by a considerable margin. In this section, we further provide some interpretations of the performance results.

- Protocols using a more comprehensive allocation strategy perform better. For example, in the DSA++, DTDMA/PR, and SCAMA protocols, the prioritization process which takes into account the important factors such as throughput required and urgency. The SCAMA protocol outperforms the others because one more important factor, namely the channel condition, is also considered.

- Capture effect does not help improving the performance of protocols. This indicates that the performance bottleneck is in the allocation of information slots, rather than the resolution of requests contention.

- In view of the superior performance of both the DTDMA/VR and SCAMA protocols, using a variable throughput adaptive physical layer seems to be a mandatory consideration in future MAC protocol design. The rationale is that as burst errors are unavoidable and are time-varying in a rather predictable manner (e.g., Rayleigh), exploiting the channel condition using a dynamic approach (i.e., varying the instantaneous 


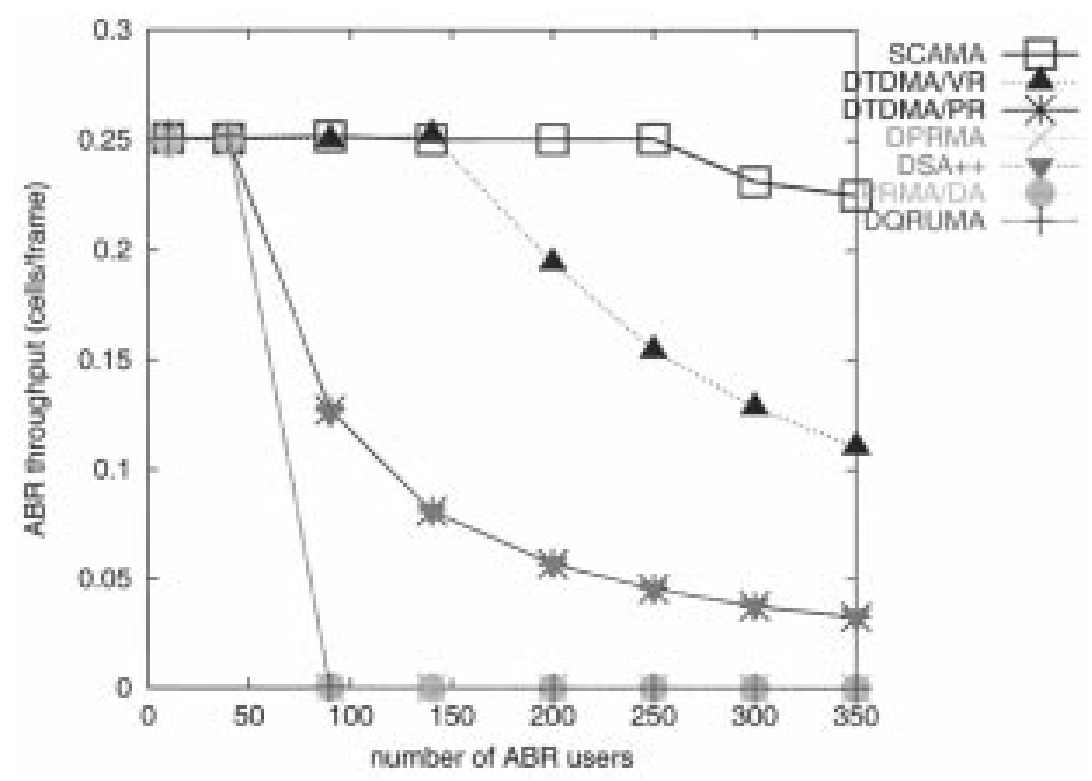

(a)

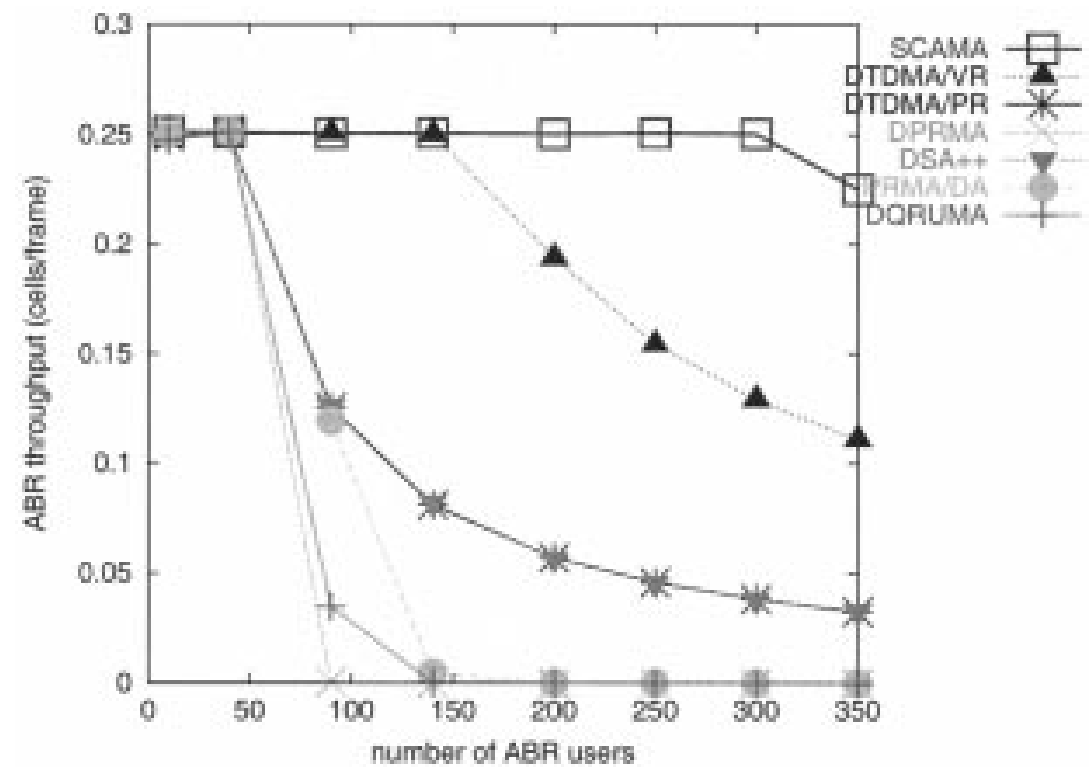

(b)

Fig. 17. Performance of the protocols for ABR users (throughput versus traffic load; five VBR users).

throughput), instead of a static one (e.g., incorporating large amount of FEC statically and relying on ARQ), is much more efficient.

- A CBR terminal may experience a deep fading for a long time when it is affected by shadowing. In the other protocols (including DTDMA/VR), bandwidth allocation in the MAC layer is carried out regardless of the current channel condition as detected in the physical layer. Thus, information slots could also be allocated to such a user and the transmitted packets will be very likely lost due to the poor channel condition. In other words, assigned slots are simply wasted. This kind of wasteful allocation is avoided in the SCAMA protocol.

- Selection diversity is implicitly incorporated in the SCAMA protocol. Through the priority-based assignment process, every frame is packed with a selected group of information cells with good channel states. Thus, the effective delivered throughput per frame achieved in SCAMA can be much higher than that in DTDMA/VR and other protocols. In SCAMA, a large number of transmission requests are collected first before allocation of information slots. From the collection of requests, there is a high likelihood that a sufficient number of requests with good channel states can be selected to fully utilize the information slots in an effective manner (i.e., high throughput). For those requests with poor instantaneous channel states, their transmissions are deferred until when the CSI improves or the deadlines are approaching. By contrast, in the DTDMA/VR and DQRUMA protocols, requests are served in a first-come-first-serve manner due to the traditional strategy of immediately assigning slots upon successful receipt of requests. Thus, the channel 


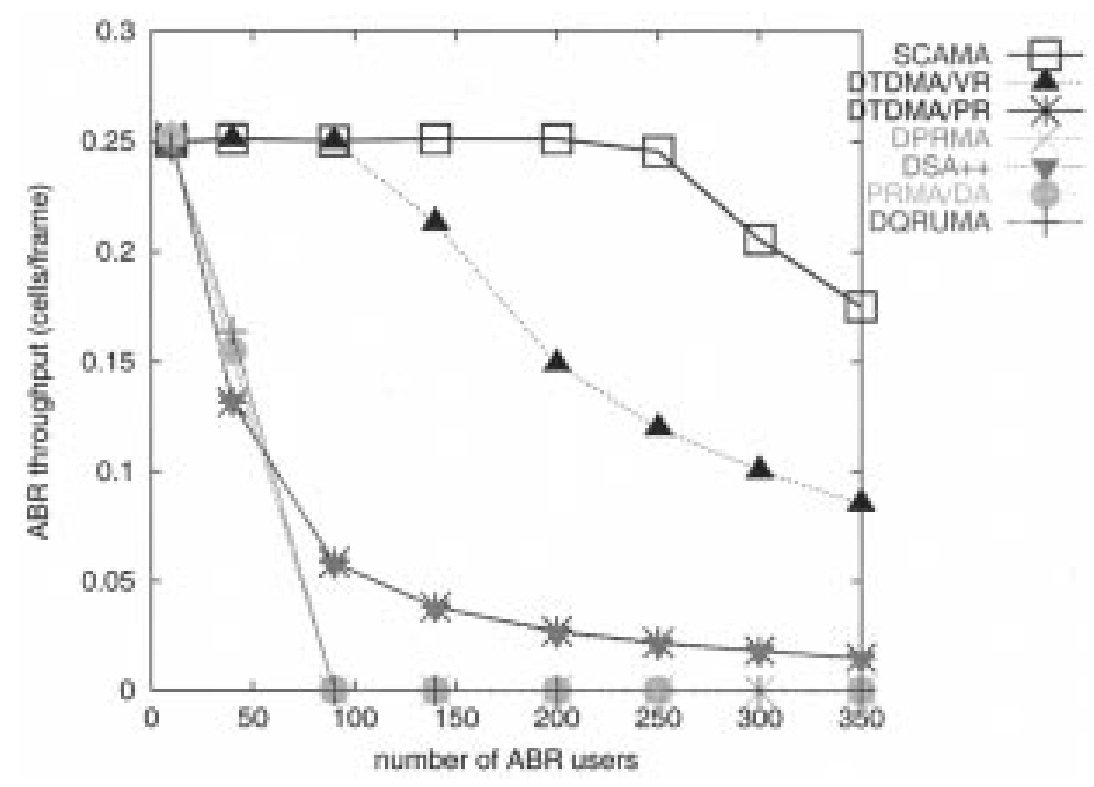

(a)

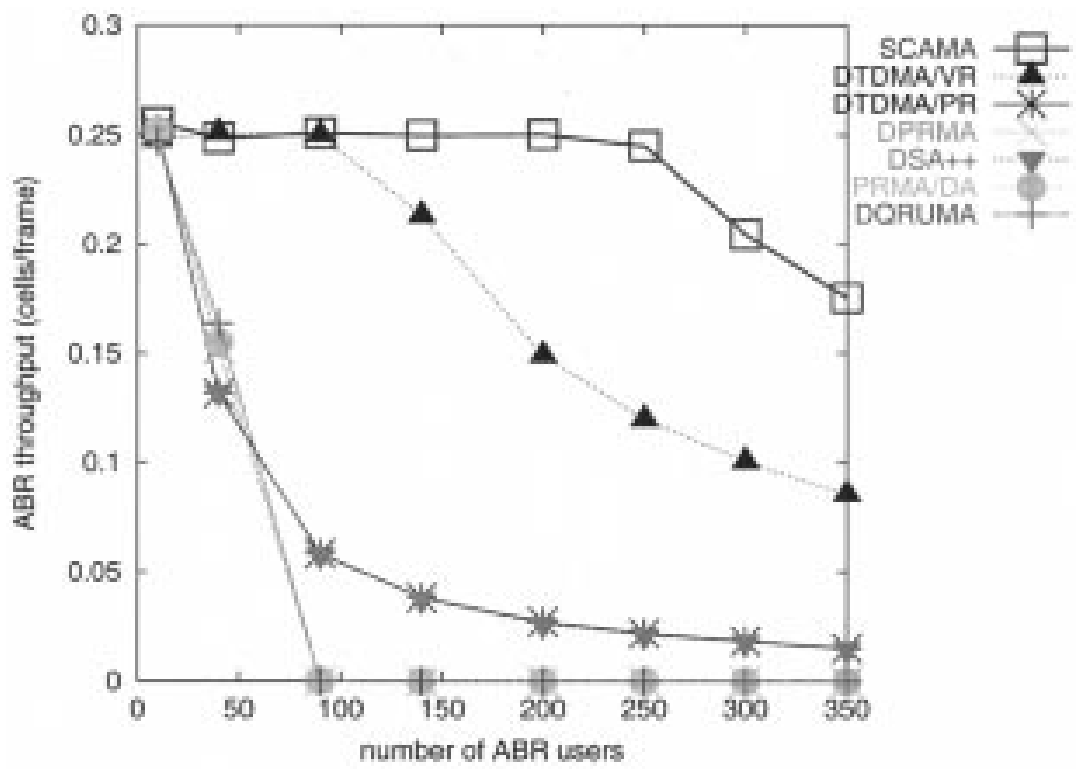

(b)

Fig. 18. Performance of the protocols for ABR users (throughput versus traffic load; ten VBR users).

states of such requests are highly diverse and, most importantly, some requests with bad channel states (hence very low throughput) are also served, whereby causing inefficient the bandwidth utilization. For example, a CBR terminal may experience a very good CSI for a long time (out of shadowing). In protocols without considering CSI in the prioritization process (DTDMA/PR) or those even without explicit prioritization (DTDMA/VR and DQRUMA), this user, however, may fail to successfully transmitted a request to the base station, probably because of excessive collisions in the request phase. In comparison, the SCAMA protocol gathers a large number of requests through successive frames, and allocate time slots to the users that can use the system bandwidth more effectively. Thus, the likelihood of "missing" a user with good channel state is much lower and the utilization of bandwidth is therefore higher.

\section{CONCLUSION}

We have presented an extensive performance comparison of seven recently proposed MAC protocols, namely SCAMA, DPRMA, DTDMA/VR, DTDMA/PR, PRMA/DA, DSA++, and DQRUMA. Using our proposed taxonomy of TDMA-based MAC protocols, these FDD based protocols are carefully selected such that they are devised based on rather orthogonal designed philosophies. The protocols are extensively evaluated for test cases with and without capture. Our simulation results reveals that using a variable-throughput physical layer (in the SCAMA and DTDMA/VR protocols) can help reducing 
voice packet dropping due to transmission errors. Finally, in view of the fact that the SCAMA protocol outperforms the other six protocols in terms of CBR/VBR cell-loss rate, ABR throughput and delay, all by a considerable margin, the knowledge of the channel condition reported to the MAC layer by the physical layer (in the SCAMA protocol) is indeed a very useful component in achieving even higher performance in a wireless communication system where burst errors are the norm rather than exception.

\section{ACKNOWLEDGMENT}

The authors would like to thank the reviewers for their constructive and insightful comments which have improved the paper significantly.

\section{REFERENCES}

[1] J. C. Ambak and W. van Blitterswijk, "Capacity of slotted ALOHA in Rayleigh fading channels," IEEE J. Select. Areas Commun., vol. SAC-5, pp. 261-268, Feb. 1987.

[2] F. Bauchot et al., "MASCARA: A MAC protocol for wireless ATM," in Proc. ACTS Mobile Summit '96, Nov. 1996, pp. 17-22.

[3] J.-C. Chen, K. M. Sivalingam, and R. Acharya, "Comparative analysis of wireless ATM channel access protocols supporting multimedia traffic," Mobile Networks Applicat., vol. 3, pp. 293-306, 1998.

[4] D. A. Dyson and Z. J. Haas, "A dynamic packet reservation multiple access scheme for wireless ATM," Mobile Networks Applicat., vol. 4, pp. 87-99, 1999.

[5] D. J. Goodman, R. A. Valenzuela, K. T. Gayliard, and B. Ramamurthi, "Packet reservation multiple access for local wireless communications," IEEE Trans. Commun., vol. 37, pp. 885-890, Aug. 1989.

[6] J. Gruber and L. Strawczynski, "Subjective effects of variable delay and speech clipping in dynamically managed voice systems," IEEE Trans. Commun., vol. COM-33, pp. 801-808, Aug. 1985.

[7] M. J. Karol, Z. Liu, and K. Y. Eng, "Distributed-queueing requests update multiple access (DQRUMA) for wireless packet (ATM) networks," in Proc. ICC'95, vol. 2, Seattle, WA, June 1995, pp. 1224-1231.

[8] M. Kawagishi, S. Sampei, and N. Morinaga, "A novel reservation TDMA based multiple access scheme using adaptive modulation for multimedia wireless communication systems," in Proc. Vehicular Technology Conf.'98, vol. 1, Ottawa, ON, Canada, May 1998, pp. 112-116.

[9] J. G. Kim and I. Widjaja, "PRMA/DA: A new media access control protocol for wireless ATM," in Proc. ICC'96, vol. 1, Dallas, TX, June 1996 pp. 240-244.

[10] T. V. Lakshman, A. Ortega, and A. R. Reibman, "VBR video: Tradeoff and potentials," Proc. IEEE, vol. 86, pp. 952-973, May 1998.

[11] V. K. N. Lau, "Performance of variable rate bit-interleaved coding for high bandwidth efficienty," in Proc. Vehicular Technology Conf.'00, vol. 3, Tokyo, Japan, May 2000, pp. 2054-2058.

[12] V. K. N. Lau and Y.-K. Kwok, "On channel adaptive multiple access control with queued transmission requests for wireless ATM," in Proc. 2000 IEEE Conf. High Performance Switching and Routing, Heidelberg, Germany, June 2000, pp. 473-481.
[13] D. Petras and A. Krämling, "Wireless ATM: Performance evaluation of a DSA++ MAC protocol with fast collision resolution by a probing algorithm," Int. J. Wireless Inform. Networks, vol. 4, no. 4, pp. 215-223, Oct. 1997.

[14] X. Qiu, V. O. K. Li, and J.-H. Ju, "A multiple access scheme for multimedia traffic in wireless ATM," ACM/Baltzer Mobile Networks Applicat., vol. 1, pp. 259-272, 1996.

[15] D. Raychaudhuri, L. J. French, R. J. Siracusa, S. K. Biswas, R. Yuan, P. Narasimhan, and C. A. Johnston, "WATMnet: A prototype wireless ATM system for multimedia personal communication," IEEE J. Select. Areas Commun., vol. 15, pp. 83-95, Jan. 1997.

[16] J. Sanchez, R. Martinez, and M. W. Marcellin, "A survey of MAC protocols proposed for wireless ATM," IEEE Network, vol. 11, pp. 52-62, Nov./Dec. 1997

[17] A. Urie, M. Streeton, and C. Mourot, "An advanced TDMA mobile access system for UMTS," in Proc. IEEE PIMRC'94, vol. 2, Amsterdam, The Netherlands, Sept. 1994, pp. 685-690.

[18] M. D. Yocoub, Foundations of Mobile Radio Engineering, 2nd ed. New York: McGraw-Hill, 1986.

[19] H. Zhang, "Service disciplines for guaranteed performance service in packet-switching networks," Proc. IEEE, vol. 83, pp. 1374-1396, Oct. 1995.

Yu-Kwong Kwok (S'94-M'95) received the B.Sc. degree in computer engineering from the University of Hong Kong in 1991 and the M.Phil. and Ph.D. degrees in computer science from the Hong Kong University of Science and Technology in 1994 and 1997, respectively.

$\mathrm{He}$ is an Assistant Professor in the Department of Electrical and Electronic Engineering at the University of Hong Kong. Before joining the University of Hong Kong, he was a Visiting Scholar for one year in the parallel processing laboratory at the School of Electrical and Computer Engineering at Purdue University, West Lafayette, IN. His research interests include mobile computing, wireless communication protocols, software support for parallel and distributed computing, heterogeneous cluster computing, and distributed multimedia systems.

Dr. Kwok is a member of the IEEE Computer Society and the ACM.

Vincent K. N. Lau received the B.Eng. degree in electrical and electronic engineering from the University of Hong Kong in 1993 and the Ph.D. degree in electrical engineering from the University of Cambridge, U.K., in 1997.

$\mathrm{He}$ is an Assistant Professor in the Department of Electrical and Electronic Engineering, University of Hong Kong. Before joining the University of Hong Kong, he was a Member of the Technical Staff at Lucent Technologies, where he worked in the design and development of third generation wide-band CDMA [cdma2000]. He was involved in the standardization, design of tools and algorithms, as well as prototype development for the $3 \mathrm{G}$ Base Station. His research interests include information theory, adaptive channel coding and modulation, power control and CREST factor control algorithms, and multiple-access protocols for $3 \mathrm{G}$ wide-band CDMA systems. 